\begin{tabular}{|c|l|}
\hline Title & Bent rectangles as viscosity solutions over a circle \\
\hline Author(s) & GIGA, Y OSHIKAZU; Gorka, Przemyslaw; Rybka, Piotr \\
\hline Citation & Hokkaido University Preprint Series in Mathematics, 1062, 1-35 \\
\hline Issue Date & 201410-28 \\
\hline DOI & 10.14943/84206 \\
\hline Doc URL & http://hdl.handle.net/2115/69866 \\
\hline Type & bulletin (article) \\
\hline File Information & pre1062.pdf \\
\hline
\end{tabular}

Instructions for use 


\title{
Bent rectangles as viscosity solutions over a circle
}

\author{
Yoshikazu Giga $^{1}$, Przemysław Górka ${ }^{2}$, Piotr Rybka ${ }^{3}$ \\ ${ }^{1}$ Graduate School of Mathematical Sciences \\ University of Tokyo \\ Komaba 3-8-1, Tokyo 153-8914, Japan \\ e-mail: labgiga@ms.u-tokyo.ac.jp \\ ${ }^{2}$ Department of Mathematics and Information Sciences \\ Warsaw University of Technology \\ pl. Politechniki 1, 00-661 Warsaw, Poland \\ e-mail: P.Gorka@mini.pw.edu.pl \\ ${ }^{3}$ Institute of Applied Mathematics and Mechanics, Warsaw University \\ ul. Banacha 2, 07-097 Warsaw, Poland \\ fax: +(48 22) 554 4300, e-mail: rybka@mimuw . edu .pl
}

October 27, 2014

\begin{abstract}
We study the motion of the so-called bent rectangles by the singular weighted mean curvature. We are interested in the curves which can be rendered as graphs over a smooth onedimensional reference manifold. We establish a sufficient condition for that. Once we deal with graphs we can have the tools of the viscosity theory available, like the Comparison Principle. With its help we establish uniqueness of variational solutions constructed by the authors [18]. In addition, we establish a criterion for the mobility coefficient guaranteeing vertex preservation.
\end{abstract}

\section{Introduction}

An important part of a model of a single crystal growing in the atmosphere or from a solution is the Gibbs-Thomson law on a crystal surface, see [23], [24], [31], [32],

$$
\beta V=\kappa_{\gamma}+\sigma .
$$

This equation relates the velocity of the advancing surface $V$ to its weighted mean curvature $\kappa_{\gamma}$ and the amount of matter $\sigma$, where $\beta>0$ denotes the mobility depending on the orientation of the surface. The interpretation of $\sigma$ depends upon the particular phenomenon we discuss. The meaning of symbols used in (1.1) is explained in Section 2.1 We shall see then, that from the point of view of differential equations (1.1) is the weighted mean curvature flow with forcing.

In the full model the Gibbs-Thomson relation, (1.1), is coupled to the diffusion equation for supersaturation $\sigma$ (temperature, pressure etc). The literature is abundant since this topic 
has been studied for a long time, a biased sample is: [1], [10] [12], [27], [28], [29], [30] and references therein. Any realistic modeling attempt should take into account, see [3]:

1) lack of smoothness of the growing surface;

2) lack of smoothness of the surface energy function appearing in the definition of the wmc $\kappa_{\gamma}$. If we, in addition, expect a qualitative analysis of solutions, then there is probably no theory available.

We studied, in [18] (see also references therein), the evolution of bent rectangle by (1.1). Deformed rectangles are approximate cross sections of columnar ice crystals found in Nature, as this is seen from Nakaya diagram, see [26]. We established, in [18], the existence of variational solutions to (1.1) for bent rectangles (see the definition in Section 2 below), when $\sigma$ is a given function conforming to the so-called Berg effect, see [9], [19], [25]. In the next section, we provide more details.

Our existence result treats the situation at the onset of facet breaking or right after it, in other words, the initial data are not general. In the simplest case we have three facets on each side of the bent rectangle. We recall that by a facet we understand a flat part of $\Gamma$ whose normal vector is a singular direction of $\gamma$.

The variational solutions are 'semi-explicit' and this makes them easy to analyze. This also becomes a drawback in more complicated situations. This is exactly the reason why we consider, in [18], only a limited class of initial conditions. The difficulty is related to a variable character of the endpoints of the facets. This may be explained as follows. Once we write (1.1) in a local coordinate system, then we obtain a Hamilton-Jacobi equation with a free boundary - the facet endpoints. The free boundary is either a 'shock wave' or a 'rarefaction wave', depending upon the data. We have a strong feeling that growing complexity of cases, we study, calls for a new, more general tool.

Moreover, the uniqueness result in [18] is limited to a special configuration of the data. Here, we want to lift it, but only for bent rectangles, which are graphs of a piecewise $C^{1}$ functions over a smooth reference manifold. We present a geometric condition on the data which guarantees that such a manifold exists. This is done in Theorem 3.1 in Section 3. Once we reach that goal, we concentrate on showing that the variational solutions are indeed viscosity solutions in the sense of [16], developed for equations like

$$
u_{t}=a\left(u_{x}\right)\left(\left(W_{p}\left(u_{x}\right)\right)_{x}+\sigma\right) \quad(x, t) \in \Omega \times(0, T)
$$

augmented with periodic boundary conditions as well as initial data. In this equation $W$ is a convex, continuous and piece-wise $C^{2}$ function.

However, we can only show that a profile function $u$ (see Definition 4.3 for a rigorous statement) of a family $\{\Gamma(t)\}_{t \in[0, T)}$ of bent rectangles satisfies equation like (1.2), but the coefficient $a$ depends not only on $u_{x}$ but also on $u$ and $x$ in a non-trivial way, as well as $W$ depends on $x$,

$$
u_{t}=a\left(u_{x}, u, x\right)\left(\left(W_{p}\left(u_{x}, x\right)\right)_{x}+\sigma\right) \quad(x, t) \in \Omega \times(0, T),
$$

see Theorem 3.2. This is not an obstacle for introducing the notion of viscosity solutions like in [16], but we have to check if the Comparison Principle, [16, Theorem 7] is still valid.

We show that the variational solutions to (1.1) constructed in [18] are viscosity solutions. This is done in Section 4. An easy part of this proof is done in [16, Section 5]. Here, we concentrate on the behavior of the vertex. In [18], we assumed that the verteces of $\Gamma(t)$ are defined 
as intersection of facets. Here, we look more closely at this issue pointing to the behavior of the kinetic coefficient, which is crucial to solve this issue. We formulate a condition on $\beta$ which is necessary and sufficient for the profile function to be a viscosity solution to an equation like (1.2), see (4.16) in Theorem 4.2. We notice that a similar problem was studied in [15], it is not surprising that the condition on the mobility coefficient $1 / \beta$, see [15, eq. (4.5)] looks similar to (4.16). The main difference is that we consider here a non-constant $\sigma, W$ depends on $x, \Gamma(t)$ is from a restricted class of curves and we have a slightly different equation (??).

As we mentioned, we have to deal with (1.3). Thus, we have to establish a Comparison Principle for viscosity solutions of (1.3). This is done by re-working the proof presented in [16]. The basic idea is the doubling variables. The Comparison Principle is proved in Section 5. It implies uniqueness of viscosity solutions, hence variational ones.

So far, we presented the content of this paper without drawing a bigger picture. Let us mention that despite efforts of many researchers, see references mentioned earlier, there is no complete study of the (two-phase or one-phase) modified Stefan problem with Gibbs-Thomson relation (1.1), when the interface is not smooth and the interfacial energy function $\gamma$ is singular. On the other hand, we have a successful attempt by [3] to solve the complete Stefan problem with Gibbs-Thomson law numerically. Their simulations are capable to capture quite different qualitative behavior corresponding to different physical parameters. However, their work is based on careful regularization of the surface energy density $\gamma$.

It is a challenge to justify theoretically the pictures obtained in [3]. A way to cope with this problem is to study (1.1) for non-smooth $\gamma$, also when $\sigma$ is given. We concentrate on an aspect of this task in the present paper.

\section{Preliminaries}

\subsection{The setting of variational solutions}

In general, solving (1.1) is challenging. In our earlier papers we restricted our attention to bent rectangles. Their definition is recalled below. The reason for considering such curves is the following. The Wulff shape, $W_{\gamma}$, of the energy density function $\gamma$ given by (2.3), i.e. the curve minimizing the energy functional $E(\Gamma)=\int_{\Gamma} \gamma(\mathbf{n}) d \mathcal{H}^{1}$ under the area constraint, is a rectangle. A bent rectangle is a kind of a small perturbation (in the $C^{0}$-topology) of a scaled $W_{\gamma}$, resulting from overgrowing $W_{\gamma}$ in a diffusion field $\sigma$.

We explain the basic notions used in this paper. In equation (1.1) $V$ denotes the normal velocity of curve $\Gamma, \beta=\beta(\mathbf{n})$ is the kinetic coefficient. The driving term $\sigma=\sigma\left(x_{1}, x_{2}, t\right)$ in our setting is given, it may be interpreted as supersaturation, temperature etc. We assume that $\sigma$ satisfies the so-called Bergs effect, see [9] for the experimental evidence. Analytically, this may be written as

$$
x_{i} \frac{\partial \sigma}{\partial x_{i}}\left(x_{1}, x_{2}\right)>0
$$

for $x_{i} \neq 0$. We consider only $\Gamma(t)$ which are bent rectangles, introduced in [21]. For the sake of self-consistency, we recall the definition below.

Formally, we define $\kappa_{\gamma}$ as

$$
\kappa_{\gamma}=-\operatorname{div}\left(\left.\nabla_{\xi} \gamma(\xi)\right|_{\xi=\mathbf{n}}\right)
$$


where

$$
\gamma\left(\tilde{p}_{1}, \tilde{p}_{2}\right)=\gamma_{\Lambda}\left|\tilde{p}_{1}\right|+\gamma_{R}\left|\tilde{p}_{2}\right| .
$$

Then, indeed the normals to the facets of $\Gamma(t)$ are singular directions of $\gamma$. The variational definition of $\kappa_{\gamma}$ will be recalled in subsection 2.3

\subsection{Bent rectangles}

We shall call a Lipschitz closed curve $\Gamma$ a bent rectangle (see $[22, \S 2])$ if the following conditions are satisfied:

There exist even, Lipschitz continuous functions $d^{R}, d^{\Lambda}: \mathbb{R} \rightarrow \mathbb{R}_{+}$, which are nondecreasing for positive arguments and there are positive numbers $L_{1}, R_{1}$ such that

$$
d^{\Lambda}\left(L_{1}\right)=R_{1}, \quad d^{R}\left(R_{1}\right)=L_{1} .
$$

In addition, $d^{\Lambda}$ is constant in a neighborhood of zero and $L_{1}$ (respectively, $d^{R}$ is constant in a neighborhood of zero and $R_{1}$ ). Furthermore,

$$
\Gamma=\partial\left\{\left(x_{1}, x_{2}\right):\left|x_{1}\right| \leq d^{\Lambda}\left(x_{2}\right),\left|x_{2}\right| \leq d^{R}\left(x_{1}\right)\right\} .
$$

We shall call $d^{R}, d^{\Lambda}$ a pair of admissible functions.

We shall call the points $\left( \pm R_{1}, \pm L_{1}\right)$ verteces of $\Gamma$. Thus, after we set

$$
\begin{aligned}
& S_{\Lambda}^{ \pm}=\left\{\left(x_{1}, x_{2}\right) \in \Gamma: x_{1}= \pm d^{\Lambda}\left(x_{2}\right), x_{2} \in\left[-L_{1}, L_{1}\right]\right\}, \\
& S_{R}^{ \pm}=\left\{\left(x_{1}, x_{2}\right) \in \Gamma: x_{2}= \pm d^{R}\left(x_{1}\right), x_{1} \in\left[-R_{1}, R_{1}\right]\right\},
\end{aligned}
$$

we notice that the graphs of $\pm\left. d^{\Lambda}\right|_{\left[-L_{1}, L_{1}\right]}, \pm\left. d^{R}\right|_{\left[-R_{1}, R_{1}\right]}$ make up the whole $\Gamma(t)$, i.e.

$$
\Gamma=S_{R}^{-} \cup S_{R}^{+} \cup S_{\Lambda}^{-} \cup S_{\Lambda}^{+}
$$

We will call $S_{R}^{ \pm}$and $S_{\Lambda}^{ \pm}$the sides of $\Gamma(t)$. Verteces of $\Gamma$ are the intersections $S_{R}^{ \pm} \cap S_{\Lambda}^{ \pm}$.

Since $S_{\Lambda}^{-}$(resp. $S_{R}^{-}$) is the symmetric image of $S_{\Lambda}^{+}$(resp. $S_{R}^{+}$), we will subsequently drop the superscript, while considering only $S_{\Lambda}^{+}\left(\right.$resp. $\left.S_{R}^{+}\right)$.

\subsection{Singular curvature $\kappa_{\gamma}$ and the notion of a variational solution}

The curvature, $\kappa_{\gamma}$, appearing in (1.1) is defined by (2.2). In this formula $\mathbf{n}$ is the outer normal to $\Gamma$ and $\gamma$ is a surface energy function (2.3). Here, the fundamental problem is apparent: $\nabla \gamma(\mathbf{n})$ is not defined on bent rectangles on sets of positive $\mathcal{H}^{1}$-measure. We resolved this issue by replacing $\nabla \gamma$ by $\partial \gamma$, which is always well-defined, because of the convexity of $\gamma$. The subdifferential coincides with $\left\{\nabla \gamma\left(x_{0}\right)\right\}$ when $\gamma$ is differentiable at $x_{0}$.

Since in general, $\partial \gamma$ is not a singleton, this leaves us with a necessity to select the proper Cahn-Hoffman vector field $\xi(x) \in \partial \gamma(\mathbf{n}(x))$. We note that this task is not obvious, where $\partial \gamma$ is not a singleton. This is why we use a variational principle as in [21], [22]. A similar approach was first introduced by [13] for graph-like solutions and was developed in several ways by the authors of [2] and [6]-[8], [4], [5]. 
We impose quite natural constraints on $\xi$, see [21],

$$
\operatorname{div}_{S} \xi \in L^{2}\left(S_{i}\right), \quad i=R, \Lambda .
$$

This implies that $\xi \cdot \nu$ has a trace, where $\nu \in T_{x} S_{i}$ is a normal vector to $S_{i}, i=R, \Lambda$. If we combine it with

$$
\partial \gamma\left(\mathbf{n}_{R}\right) \cap \partial \gamma\left(\mathbf{n}_{\Lambda}\right)=\{p\}
$$

where $\mathbf{n}_{R}=(0,1), \mathbf{n}_{\Lambda}=(1,0)$, then we see that $\xi$ satisfies a boundary condition

$$
\left.\xi\right|_{\text {vertex }}=p \text {. }
$$

The necessity of selecting $\xi$ implies that in order to define a solution to (1.1), we need to specify not only a curve $\Gamma(t)$ but also $\xi(t, \cdot)$. After [21], we recall the notion of solution. Namely, by $a$ solution to (1.1) we call a family of couples $(\Gamma(t), \xi(t)), t \in[0, T)$, such that for some $T>0$, the following conditions are satisfied:

(a) For each $t \in[0, T)$ the curve $\Gamma(t)$ is a bent rectangle and $d^{\Lambda}, d^{R}$ are continuous functions of its arguments, for each $x, d^{j}(\cdot, x), j=\Lambda, R$ are Lipschitz continuous and for each $t \in[0, T)$, functions $d^{j}(t, \cdot), j=\Lambda, R$ are admissible;

(b) $\xi: \bigcup_{t \in[0, T)}\{t\} \times \Gamma(t) \rightarrow \mathbb{R}^{2}$ is at each time instant a Cahn-Hoffman vector. If $M:=$ $\sup _{t \in[0, T)} \max \left\{L_{1}(t), R_{1}(t)\right\}+1$, and if for $j=\Lambda, R$, we set

$$
\begin{gathered}
\tilde{\xi}^{R}(t, x) \in \begin{cases}\left\{\left(-\gamma\left(\mathbf{n}_{\Lambda}\right), \gamma\left(\mathbf{n}_{R}\right)\right)\right\} & x \in\left[-M,-R_{1}(t)\right], \\
\left\{\xi\left(t,\left(x, d^{R}(t, x)\right)\right)\right\} & x \in\left[-R_{1}(t), R_{1}(t)\right], \\
\left\{\left(\gamma\left(\mathbf{n}_{\Lambda}\right), \gamma\left(\mathbf{n}_{R}\right)\right)\right\} & x \in\left[R_{1}(t), M\right] ;\end{cases} \\
\tilde{\xi}^{\Lambda}(t, x) \in \begin{cases}\left\{\left(-\gamma\left(\mathbf{n}_{\Lambda}\right),-\gamma\left(\mathbf{n}_{R}\right)\right)\right\} & x \in\left[-M,-L_{1}(t)\right], \\
\left\{\xi\left(t,\left(d^{\Lambda}(t, x), x\right)\right)\right\} & x \in\left[-L_{1}(t), L_{1}(t)\right], \\
\left\{\left(-\gamma\left(\mathbf{n}_{\Lambda}\right), \gamma\left(\mathbf{n}_{R}\right)\right)\right\} & x \in\left[L_{1}(t), M\right] ;\end{cases}
\end{gathered}
$$

then we assume that $t \mapsto \tilde{\xi}^{j}(t, \cdot) \in L^{\infty}\left(0, T ; L^{2}(-M, M)\right), j=\Lambda, R$;

(c) Equation (1.1) is satisfied in the $L^{2}$ sense for a.e. $t \geq 0$ after interpreting $\kappa_{\gamma}$ as $-\operatorname{div}{ }_{S} \xi$.

In principle, the Cahn-Hoffman vector depends upon time $t$ and $x=\left(x_{1}, x_{2}\right) \in \Gamma(t)$. However, we shall frequently suppress $t$ and write $\xi(x)$, when the meaning of the spacial argument is clear from the context, e.g. on the sides. We also distinguished variational solutions based on a specific way to select $\xi$. For this purpose, we introduce functionals,

$$
\mathcal{E}_{j}(\xi)=\frac{1}{2} \int_{S_{j}}\left|\sigma-\operatorname{div}_{S} \xi\right|^{2} \mathcal{H}^{1}, \quad j=R, \Lambda .
$$

Their natural domains are the sets of Cahn-Hoffman vectors, satisfying all the above constraints,

$$
\begin{aligned}
& \mathcal{D}_{\Lambda}=\left\{\xi \in L^{\infty}\left(S_{\Lambda}\right): \xi(x) \in \partial \gamma(\mathbf{n}(x)), \operatorname{div}_{S} \xi \in L^{2}\left(S_{\Lambda}\right), \text { (2.6) holds }\right\}, \\
& \mathcal{D}_{R}=\left\{\xi \in L^{\infty}\left(S_{R}\right): \xi(x) \in \partial \gamma(\mathbf{n}(x)), \operatorname{div}_{S} \xi \in L^{2}\left(S_{R}\right), \text { (2.6) holds }\right\},
\end{aligned}
$$

where

$$
\xi\left( \pm R_{1}, \pm L_{1}\right) \in \partial \gamma\left( \pm \mathbf{n}_{\Lambda}\right) \cap \partial \gamma\left( \pm \mathbf{n}_{R}\right) .
$$

We recall (see also [22] for a discussion of this notion) that $\{(\Gamma(t), \xi(t))\}, t \in[0, T)$, a solution to (1.1), is called a variational solution if for each $\left.t \in[0, T) \xi\right|_{S_{j}}(t) \in L^{2}\left(S_{j}\right)$ is a solution to

$$
\mathcal{E}_{j}(\xi)=\min \left\{\mathcal{E}_{j}(\zeta): \zeta \in \mathcal{D}_{j}\right\}, \quad j=R, \Lambda
$$




\subsection{Facets}

Let us consider an open line segment $I$ in the plane, i.e. $I=(a, b) \equiv\{x=a t+b(1-t), t \in$ $(0,1)\}$, where $a, b \in \mathbb{R}^{2}$. We shall say that $I \subset \Gamma$, having a normal equal to $\mathbf{n}_{\Lambda}$ or $\mathbf{n}_{R}$, is $a$ faceted region of $\Gamma$, also called $a$ facet, if it is maximal (with respect to inclusion) and it satisfies

$$
\left.\left(\sigma-\operatorname{div}_{S} \xi\right)\right|_{I}=\text { const. }
$$

where $\xi$ is a solution to (2.7).

We keep in mind that, $S_{\Lambda}^{ \pm}(t)$ and $S_{R}^{ \pm}(t)$ are graphs, e.g. $S_{R}^{+}$is the image of segment $[-R(t), R(t)]$ under the function

$$
x \mapsto\left(x, d^{R}(t, x)\right)=: \tilde{d}^{R}(t, x) .
$$

Frequently, it is more convenient to work with the inverse image of a faceted region $I$, i.e. $(\alpha, \beta)=\tilde{d}^{-1}(I)$. We stress that this definition permits $S_{j}^{ \pm}(t), j=R, \Lambda$ being a line segment which has more than one faceted region.

Let us make a few comments on the definition of solutions. As it is stated, the variational solutions has its limitations. First of all, it does not permit handling the curved parts, when the data are not of $C^{1}$-class. We know that the methods of viscosity solutions for Hamilton-Jacobi equations are appropriate tools, see [11]. Secondly, the position of the verteces are defined as intersections of facets, while the vertex preservation property should be deduced from the equations.

We solve these issues with the help of viscosity solutions introduces in Section 4. However, there is a price: we have to restrict slightly the class of admissible initial data to such that satisfy the geometric condition (3.3).

\subsection{Existence of variational solutions}

Here, we recall the main existence result, see [18]. We restrict our attention to such variational solutions $(\Gamma, \xi)$ of (1.1) that each facet $S_{j}$ has exactly three faceted regions, whose pre-images are:

$$
\left(-L_{1},-l_{1}\right), \quad\left(-l_{0}, l_{0}\right), \quad\left(l_{1}, L_{1}\right), \quad\left(-R_{1},-r_{1}\right), \quad\left(-r_{0}, r_{0}\right), \quad\left(r_{1}, R_{1}\right) .
$$

We need to recall some notations and definitions. Initial conditions for interfaces will be denoted as follows

$$
\begin{aligned}
& l_{0}(0)=l_{00}, \quad l_{1}(0)=l_{10}, \quad r_{0}(0)=r_{00}, r_{1}(0)=r_{10}, \\
& R_{0}(0)=R_{00}, \quad R_{1}(0)=R_{10}, \quad L_{0}(0)=L_{00}, \quad L_{1}(0)=L_{10} .
\end{aligned}
$$

Definition 2.1. We shall say that assumptions $(S)$ are satisfied if

$\left(S_{1}\right) \quad$ (conditions on $\sigma$ ) The forcing term $\sigma$ is of class $C^{2}$ and it satisfies the symmetry relation

$$
\sigma\left(t, x_{1}, x_{2}\right)=\sigma\left(t,-x_{1}, x_{2}\right), \quad \sigma\left(t, x_{1}, x_{2}\right)=\sigma\left(t, x_{1},-x_{2}\right) \text { for all } x_{i} \in \mathbb{R}, i=1,2 \text {. }
$$

and Berg's effect (2.1) 
$\left(S_{2}\right) \quad$ (conditions on $\beta$ ) Let us denote by $m^{i}(p)=\frac{\sqrt{1+p^{2}}}{\beta^{i}(p)}$ the mobility coefficient. The mobility coefficients are Lipschitz continuous, convex for $|p| \leq 1, C^{2}$-class on $\mathbb{R} \backslash\{0\}$ and

$$
m^{i}(0) \leq m^{i}(p), \quad m^{i}(p)=m^{i}(-p), \quad m^{i}(p) \leq C(1+|p|), \quad i=\Lambda, R .
$$

$\left(S_{3}\right) \quad$ (conditions on the initial curve) $d_{0}^{\Lambda}, d_{0}^{R}$ is an admissible pair of functions, which are of class $C^{2}$ on the closure of the complement of the preimages of the facets.

Let us recall that the tangency condition is satisfied at $r_{0}(t)$ if $\frac{\partial \xi}{\partial x_{1}}\left(r_{0}(t)\right)=0$. Moreover, we the statement of the Theorem below needs the following two quantities, $\Sigma_{0}^{R}$ introduced in [18, formula (2.22)]

$$
\begin{aligned}
\Sigma_{0}^{R}:= & f_{0}^{r_{0}} \sigma_{t}\left(0, y, L_{00}\right) d y-\sigma_{t}\left(0, R_{00}, l_{00}\right) \\
& +\sigma\left(0, r_{0}, L_{00}\right)\left(f_{0}^{r_{00}} \sigma_{x_{2}}\left(0, y, L_{00}\right) d y-\sigma_{x_{2}}\left(0, r_{0}, L_{00}\right)\right),
\end{aligned}
$$

and $\Sigma_{1}^{R}$ from [18, Proposition $\left.2.3(\mathrm{~b})\right]$,

$$
\begin{aligned}
\Sigma_{1}^{R}:= & f_{r_{10}}^{R_{10}} \sigma_{t}\left(0, y, L_{10}\right) d y-\sigma_{t}\left(0, r_{10}, L_{10}\right) \\
& +\sigma\left(0, r_{10}, L_{10}\right)\left(f_{r_{10}}^{R_{10}} \sigma_{x_{2}}\left(0, y, L_{10}\right) d y-\sigma_{x_{2}}\left(0, r_{10}, L_{10}\right)\right) \\
& +\frac{\dot{R}_{1}(0)}{\left(R_{10}-r_{10}\right)}\left(\sigma\left(t, R_{10}, L_{1}(0)\right)-\dot{L}_{1}(0)\right) .
\end{aligned}
$$

These two quantities determine the evolution of the facets parallel to the $x_{1}$-axis. We should define the corresponding objects, $\Sigma_{0}^{\Lambda}, \Sigma_{1}^{\Lambda}$ for the facets parallel to the $x_{2}$-axis, but for the sake of making the presentation concise we will not do so, but rather refer the reader to [18].

We are now ready to recall the main existence theorem.

Theorem 2.1. Let us suppose that, the standard set of assumptions (S) holds, in particular the initial curve $\Gamma_{0}$ is a regular bent rectangle, $l_{00}<l_{10}$ and $r_{00}<r_{10}$. We assume that the initial data fulfill conditions (a) and (b) below:

(a) One of the following conditions holds at the interface $r_{00}$ :

(i) $d_{0, x}\left(r_{00}\right)=0, \Sigma_{0}^{R}=\Sigma_{0}^{R}\left(0, r_{00}, L_{00}\right)<0$, the tangency condition holds at $r_{00}$ and

$$
\Sigma_{0}^{R}\left(0, r_{00}, L_{00}\right)+\frac{1}{2} \sigma_{x_{1}}\left(0, r_{00}, L_{00}\right) \sigma\left(0, r_{00}, L_{00}\right)<0 .
$$

(ii) $\dot{L}_{0}(0)-\sigma\left(0, r_{00}, L_{00}\right) m\left(d_{0, x}^{+}\left(r_{00}\right)\right) \neq 0$, the tangency condition is violated at $r_{00}$ and $d_{0, x}^{+}\left(r_{00}\right)>0$.

Moreover, a respective version of (i) and (ii) holds for $l_{00}$.

(b) One of the following conditions hold at interface $r_{10}$ : 
(iii) $d_{0, x}\left(r_{10}\right)=0, \Sigma_{1}^{R}>0$, the tangency condition holds at $r_{10}$ as well as a condition corresponding to (2.9) for $r_{10}$;

(iv) $\dot{L}_{1}(0)-\sigma\left(0, r_{10}, L_{10}\right) m\left(d_{0, x}^{-}\left(r_{10}\right)\right) \neq 0$, the tangency condition is violated at $r_{10}$ and $d_{0, x}^{-}\left(l_{10}\right)>0$.

Moreover, a respective version of (iii) and (iv) holds for $l_{10}$.

Then, there exists a variational solution to the following system (see [18, (2.17), (2.18)]), which is the localized version of (1.1) for bent rectangles,

$$
\begin{aligned}
& \beta_{R} \dot{L}_{0}=f_{0}^{r_{0}} \sigma\left(t, s, L_{0}\right) d s+\frac{\gamma\left(\mathbf{n}_{\Lambda}\right)}{r_{0}} \quad \text { on } \quad\left[0, r_{0}\right] \text {, } \\
& d_{t}^{R}=\sigma\left(t, x_{1}, d^{R}\right) m^{R}\left(d_{x}^{R}\right) \quad \text { on } \quad\left[r_{0}, r_{1}\right], \\
& \beta_{R} \dot{L}_{1}=f_{r_{1}}^{R_{1}} \sigma\left(t, s, L_{1}\right) d s-\frac{2 \gamma\left(\mathbf{n}_{\Lambda}\right)}{R_{1}-r_{1}} \quad \text { on }\left[r_{1}, R_{1}\right] \text {, } \\
& \beta_{\Lambda} \dot{R}_{0}=f_{0}^{l_{0}} \sigma\left(t, R_{0}, s\right) d s+\frac{\gamma\left(\mathbf{n}_{R}\right)}{l_{0}} \quad \text { on }\left[0, l_{0}\right] \text {, } \\
& d_{t}^{\Lambda}=\sigma\left(t, d^{\Lambda}, x_{2}\right) m^{\Lambda}\left(d_{x}^{\Lambda}\right) \quad \text { on } \quad\left[l_{0}, l_{1}\right], \\
& \beta_{\Lambda} \dot{R}_{1}=f_{l_{1}}^{L_{1}} \sigma\left(t, R_{1}, s\right) d s-\frac{2 \gamma\left(\mathbf{n}_{R}\right)}{L_{1}-l_{1}} \quad \text { on } \quad\left[l_{1}, L_{1}\right] \text {, }
\end{aligned}
$$

augmented with the following initial conditions,

$$
\begin{array}{r}
l_{0}(0)=l_{00}, \quad l_{1}(0)=l_{10}, \quad r_{0}(0)=r_{00}, \quad r_{1}(0)=r_{10}, \\
R_{0}(0)=R_{00}, \quad R_{1}(0)=R_{10}, \quad L_{0}(0)=L_{00}, \quad L_{1}(0)=L_{10}, \\
d^{R}\left(0, x_{1}\right)=d_{0}^{R}\left(x_{1}\right), \quad d^{\Lambda}\left(0, x_{2}\right)=d_{0}^{\Lambda}\left(x_{2}\right) .
\end{array}
$$

\section{Graphs over a smooth manifold}

Our first task is to show that there is a class of bent rectangles, which may be written as graphs over a smooth manifold. This will permit us to apply the theory developed in [16]. We want that our reference manifold $\mathcal{M}$ have two pairs of sides parallel to the axes. We show that actually we can construct such $\mathcal{M}$ for a class of bent rectangles.

Let $\mathcal{M}$ be a convex curve obtained by rounding off vertices of an octagon with two pairs of sides perpendicular to the coordinate axes. For our convenience we assume that $\mathcal{M}$ is negatively oriented and symmetric with respect to the axes. We assume that $\Phi: \mathbb{T}^{1}=\mathbb{R} /(2 \pi L \mathbb{Z}) \rightarrow \mathbb{R}^{2}$ is a smooth, $2 \pi L$-periodic, arclength parametrization of this manifold. Thus, in particular, $\Phi_{s}(s)=: \tau(s)$ is the unit tangent and we denote the outer normal to $\mathcal{M}$ at $\Phi(s)$ by $\nu(s)$.

For such a manifold $\mathcal{M}$, we know that there exists an open set $\mathcal{U} \subset \mathbb{T}^{1} \times \mathbb{R}$ such that

$$
\mathcal{U} \ni(s, v) \mapsto \Phi(s)+\nu(s) v:=\Psi(s, v) \in \mathbb{R}^{2}
$$

is a diffeomorphism onto an open subset of $\mathbb{R}^{2}$. Moreover, convexity of $\mathcal{M}$ implies that

$$
\mathbb{T}^{1} \times(0, \infty) \subset \mathcal{U}
$$


It is obvious, that when $\Gamma$ is a convex curve in the image of $\mathcal{U}$ under $\Psi$, then there is a $2 \pi L$ periodic function $v$ such that

$$
\Gamma=\{(x, y)=\Psi(s, v(s)):=\Phi(s)+\nu(s) v(s)\} .
$$

This follows from the definition of $\Psi$. Of course, $v$ enjoys the same class of smoothness as $\Gamma$.

It is rather obvious that not for every bent rectangle we can find a reference manifold $\mathcal{M}$ so that $\Gamma$ may be written as in (3.2). For example, in our papers, [20], [17], [18], [22] we imposed the following restriction on the data, $\left|d_{0, x}^{\Lambda}\right|,\left|d_{0, x}^{R}\right|<1$. The variational solutions to (1.1) also satisfy this bound for $t<T$. On the segments of $\mathcal{M}$ parallel to the lines $y= \pm x$ the above condition is equivalent to $\left|v_{s}\right|<+\infty$. Thus, it will not be easy to lift it.

Now, we will give our rough answer to the question which bent rectangles may be represented as (3.2). It is important to have facets representable as graphs of (piecewise) linear functions over sides of $\mathcal{M}$. The reason will be explained in Theorem 3.2.

We will say that, a bent rectangle is gently bent, if it has representation (3.2), over manifold $\mathcal{M}$ which is diffeomorphic to $S^{1}$ and convex.

Theorem 3.1. (a) Let us suppose that $\Gamma$ is a bent rectangle, symmetric with respect to both coordinate axes, and such that $\left|d_{x}^{i}\right|<1$, where $i=\Lambda, R$. If condition (3.3), stated below, holds, then $\Gamma$ is gently bent. That is, there is a smooth reference manifold $\mathcal{M}$ and a function $v: \mathcal{M} \rightarrow \mathbb{R}$ such that $\Gamma$ is a graph of $v$ over $\mathcal{M}$.

(b) Let us suppose that $\{\Gamma(t)\}_{t \in[0, T)}$ is a family of bent rectangles, with admissible functions $d^{\Lambda}(\cdot, t), d^{R}(\cdot, t)$ defining them, such that: (i) $\Gamma(0)$ is gently bent, i.e. (3.3) holds; (ii) $\Gamma(0)$ is symmetric with respect to both coordinate axes, $d^{\Lambda}, d^{R} \in C([0, T], C(\mathbb{R}))$ and $r_{i}, l_{i} \in C[0, T]$, $i=0,1$. Then, there is $\epsilon>0$ such that all $\Gamma(t)$, are gently bent for $t \in[0, \epsilon)$ with the same reference manifold $\mathcal{M}$.

Remarks. 1) The proof will be constructive, but by no means exhausting all possible cases. Let us also stress that in part (b) we claim existence of the reference manifold, which is good for a family of bent rectangles while only $\Gamma(0)$ is assumed to be symmetric with respect to the axes. 2) We may explain the geometric meaning of (3.3) as follows. There are two facet adjacent to fixed vertex. We may restrict our attention to the first quadrant. Each of the curved parts of $\Gamma$ intersecting the two facets may be put into parallelograms with one pair of sides parallel to the axis the other pair parallel to $\{y=x\}$ and the arc endpoints are verteces of the parallelograms. Now, (3.3) means that there are lines parallel to $\{y=-x\}$ intersecting the two parallelograms, see Fig. 1.

Proof of Theorem 3.1. Part (a). Let us consider the following families of horizontal, $h_{a}$, vertical, $v_{b}$, diagonal, $d_{c}$, and transversal, $t_{e}$, lines in the plane,

$$
\begin{aligned}
h_{a}=\left\{\left(x_{1}, a\right): x_{1} \in \mathbb{R}\right\}, & v_{b}=\left\{\left(b, x_{2}\right): x_{2} \in \mathbb{R}\right\}, \\
d_{c}=\left\{\left(x_{1}, x_{1}+c\right): x_{1} \in \mathbb{R}\right\}, & t_{e}=\left\{\left(x_{1}, e-x_{1}\right): x_{1} \in \mathbb{R}\right\},
\end{aligned}
$$

where the parameters $a, b, c, e$ are real numbers. If $F_{i}$ is a horizontal facet (respectively, vertical), then we may find a corresponding $a_{i}$, (respectively, $b_{i}$ ), such that $F_{i}$ is contained in $h_{a_{i}}$ (respectively, in $v_{b_{i}}$ ).

If $F_{i}$ is a central facet then we can find $d_{c_{i}}$ and $t_{e_{i}}$ so that $F_{i}$ 's endpoints lie on these lines. In other words,

$$
\partial F_{i}=\left(d_{c_{i}} \cup t_{e_{i}}\right) \cap h_{a_{i}} \quad \text { or } \quad \partial F_{i}=\left(d_{c_{i}} \cup t_{e_{i}}\right) \cap v_{b_{i}} .
$$


Actually, we can find two pairs of lines $d_{c_{i}}, t_{e_{i}}$ and $d_{c_{i}^{\prime}}, t_{e_{i}^{\prime}}$ satisfying this condition. We choose $d_{c_{i}}, t_{e_{i}}$ so that their intersection $d_{c_{i}} \cap t_{e_{i}}$ is closer to the origin than $d_{c_{i}^{\prime}} \cap t_{e_{i}^{\prime}}$.

The following construction can be carried out for each vertex independently. For the sake of clarity we fix our attention on vertex in the first quadrant.

Let us also suppose that facets are numbered counter clockwise and $F_{1}$ is the central facet lying in the half-plane $\left\{x_{2}>0\right\}$. We notice that intersections of lines $h_{a_{1}}, h_{a_{12}}, d_{c_{1}}$ and $d_{c_{12}}$ determine a parallelogram, which we will call $P_{1}$. Actually, by $P_{1}$ we mean an open bounded set. Similarly, the intersections of lines $v_{a_{10}}, v_{a_{11}}, d_{c_{10}}$ and $d_{c_{11}}$ determine a parallelogram, which we will call $P_{8}$. By the same token, we construct parallelograms $P_{2}$ to $P_{7}$. Since $\left|d_{x}^{i}\right|<1$, the curved part of $\Gamma$ joining $F_{1}$ and $F_{12}$ is in $P_{1}$ and the curved part of $\Gamma$ joining $F_{10}$ and $F_{11}$ is in $P_{8}$. We may now state our crucial condition, see Fig. 1,

$$
C_{1}:=\left\{e \in \mathbb{R}: t_{e} \cap P_{1} \neq \emptyset \text { and } t_{e} \cap P_{8} \neq \emptyset\right\} \neq \emptyset \quad \text { and } \quad C_{1}=\operatorname{int} C_{1} .
$$

We notice that for any $e \in C_{1}$ there is an open set $\mathcal{U}_{e} \subset\left(a_{1}, a_{12}\right) \times\left(b_{10}, b_{11}\right)$ such that for any $a, b \in \mathcal{U}_{e}$

$$
h_{a} \cap t_{e} \in P_{1} \text { and } v_{b} \cap t_{e} \in P_{8} .
$$

Let us suppose that $e \in C_{1}$ is given, then we will select $a$ and $b$. We will present a procedure for picking $a$ while the method of choosing $b$ is essentially the same.

For any $e \in C_{1}$ line $t_{e}$ intersects $P_{1}$. Curve $\Gamma \cap P_{1}$ intersects $t_{e}$ and the intersection consists of exactly one point $A=\left(x_{A}, d^{R}\left(x_{A}\right)\right)$. If we had more points in $d^{R} \cap P_{1} \cap t_{e}$, then this would mean that, contrary to our assumption, $d^{R}$ is not monotone. Let us suppose $\{B\}=t_{e} \cap d_{c_{12}}$. We take $E:=\frac{1}{2}(A+B)$. Now, we define $a$ to be the $x_{2}$ coordinate of $E$, thus, $\{E\}=\left\{\left(x_{E}, a\right)\right\}=t_{e} \cap h_{a}$. We set,

$$
f_{1}:=\frac{1}{2}\left(x_{A}+x_{E}\right) .
$$

Now, we draw a circle $S_{\rho}^{1}(Q)$ tangent to $h_{a}$ at $\left(f_{1}, a\right)=: G$ and also tangent to $t_{e}$. We write

$$
S_{\rho}^{1}(Q) \cap t_{e}=\{K\}=\left\{\left(x_{K}, e-x_{K}\right)\right\} .
$$

Of course, $K \in P_{1}$. We call by $\Upsilon$ the convex curve formed by $h_{a} \cap\left\{x_{1} \leq f_{1}\right\}, t_{e} \cap\left\{x_{1} \geq\right.$ $\left.x_{K}\right\}$ and the arc of $S_{\rho}^{1}(Q)$ from $G$ to $K$. We see that, $\Upsilon$ is a graph of a $C^{1}$ but not a $C^{2}$ function over the $x_{1}$-axis. We may modify it to get a $C^{\infty}$ function. In order to avoid the creation of a redundant notation, we may assume that points $G$ and $K$ are joined by smooth, convex curve meeting the lines $h_{a}$ and $t_{e}$ smoothly, i.e. all derivatives agree. Let us call the curve we have just constructed by $\Upsilon_{1}$.

A similar argument will provide us with $b$ and such that $v_{b} \cap t_{e} \in P_{8}$ and a smooth curve $\Upsilon_{2}$, whose part with non-zero curvature is contained in $P_{8}$. We may assume that $\Upsilon_{1} \cap \Upsilon_{2}=\left[K, K^{\prime}\right]$, where $K^{\prime}=\left(x_{K^{\prime}}, e-x_{K^{\prime}}\right)$ and $\left[K, K^{\prime}\right]$ denotes a line segment with endpoints $K, K^{\prime}$. We take a new curve $\Upsilon_{3}$ consisting of $\Upsilon_{1} \cap\left\{0 \leq x_{1} \leq x_{K}\right\},\left[K, K^{\prime}\right]$ and $\Upsilon_{2} \cap\left\{0 \leq x_{2} \leq e-x_{K^{\prime}}\right\}$. Of course, $\Upsilon_{3}$ is smooth.

We denote by $\mathcal{R}_{i}$ the reflection with respect to $x_{i}$-axis, $i=1,2$. We finally, define $\mathcal{M}$ as follows,

$$
\mathcal{M}=\Upsilon_{3} \cup \mathcal{R}_{1} \Upsilon_{3} \cup \mathcal{R}_{2} \Upsilon_{3} \cup \mathcal{R}_{1} \mathcal{R}_{2} \Upsilon_{3}
$$

Let us suppose that $\Phi$ is an arclength parametrization of $\mathcal{M}$, such that $\Phi(0)=(0, a), \Phi\left(\frac{\pi}{2} L\right)=$ $(b, 0)$ and $\mathcal{M}$ has negative orientation. 
At this point, it is a good idea to give names to special points on $\mathcal{M}$. Besides $0<f_{1}$, defined as the $x_{1}$-coordinate of $G$, the common endpoint of the segment $\left[-f_{1}, f_{1}\right] \times\{a\}$ and smooth arc $\widehat{G K}$, we introduce points $f_{2}^{l}, f_{2}^{r}, f_{3} \in\left[0, \frac{\pi}{2} L\right)$. We set $f_{2}^{l}$ to be such that $\Phi\left(f_{2}^{l}\right)=K$. The arc $\widehat{G K}$ meets $\left[-f_{1}, f_{1}\right] \times\{a\}$ tangentially at $G$, while $K$ is the point, where $\widehat{G K}$ tangentially intersects $\left[K, K^{\prime}\right]$ at $K$. Parameter $f_{3}$ is such that $\Phi\left(\frac{\pi}{2} L-f_{3}\right)$ and $\Phi\left(\frac{\pi}{2} L+f_{3}\right]$ are endpoints of the maximal segment in $\mathcal{M}$ contained in the half plane $\left\{x_{1}>0\right\}$, parallel to the vertical axis. Moreover, $f_{2}^{r}$ is such that $\Phi\left(f_{2}^{r}\right)$ and $\Phi\left(\frac{\pi}{2} L-f_{3}\right)$ are the endpoints of a smooth arc meeting tangentially the line segments of $\mathcal{M}$. By symmetry, we may divide the rest of $\mathcal{M}$ in a similar way.

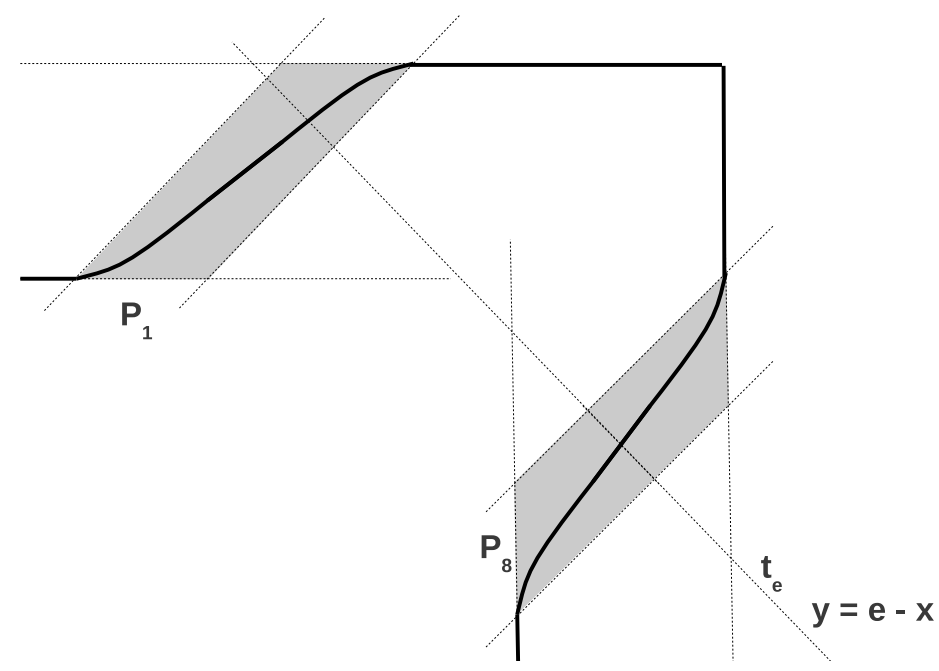

Fig. 1

By construction, $\mathcal{M}$ is smooth. It remains to check that we can find $v$, such that (3.2) holds. We assume that $s \in \mathbb{T}^{1}$ is the arclength parameter of $\mathcal{M}$ and we consider this curve with the reversed orientation. Let us recall that contrary to this choice of the orientation of $\mathcal{M}$ the parallelograms $P_{i}, i=1, \ldots, 8$ are numbered in the counter clockwise manner.

Our attention will be focused on $\Gamma \cap P_{1}$. Let us recall that

$$
\Gamma \cap P_{1}=\left\{\left(x_{1}, d^{R}\left(x_{1}\right)\right): x_{1} \in\left(r_{0}, r_{1}\right)\right\},
$$

where $\left(r_{0}, L_{0}\right)$ is one endpoint of the central facet while $\left(r_{1}, L_{1}\right)$ the endpoint in $P_{1}$ of the facet adjacent to the vertex. Since $x_{1}$ is an arclength parameter of $\mathcal{M}$ on lines parallel to the $x_{1}$-axis, then

$$
v(s):=d^{R}(s)+\left(a-L_{0}\right) \quad s \in\left[0, f_{1}\right] .
$$

Let us see that for parameters $s>f_{1}$, corresponding to $\left(\Upsilon_{1} \cap\left\{0 \leq x_{1} \leq x_{K}\right\}\right) \cup\left[K, K^{\prime}\right]$, the set $\mathbb{R}_{+} \nu(s) \cap \Gamma$ is a singleton. Thus, we can define $v$ as follows

$$
v(s)=\operatorname{dist}\left(\mathcal{M} \cap \mathbb{R}_{+} \nu(s), \Gamma \cap \mathbb{R}_{+} \nu(s)\right) .
$$

By virtue of the Implicit Function Theorem, we see that the smoothness of $v$ is inherited from d.

Finally, we can perform a similar analysis on the remaining parameters. 
Part (b). We note that if $\Gamma(t)$ is a family of bent rectangles, whose facet endpoints evolve continuously in time, then all the points on the plane we constructed in part (a) depend continuously on time. Moreover, since we can study each vertex independently, $\Gamma(t)$ need not be symmetric for $t>0$. Thus, for sufficiently small time $t \in[0, \epsilon)$ all $\Gamma(t)$ are bent rectangles.

We notice that if $\Gamma(0)$ were not symmetric with respect to the axis, then ability to perform analysis like that in part (a) need not result in a construction of a reference manifold for geometric reasons. We would need a condition like (3.3) for each vertex of $\Gamma(0)$.

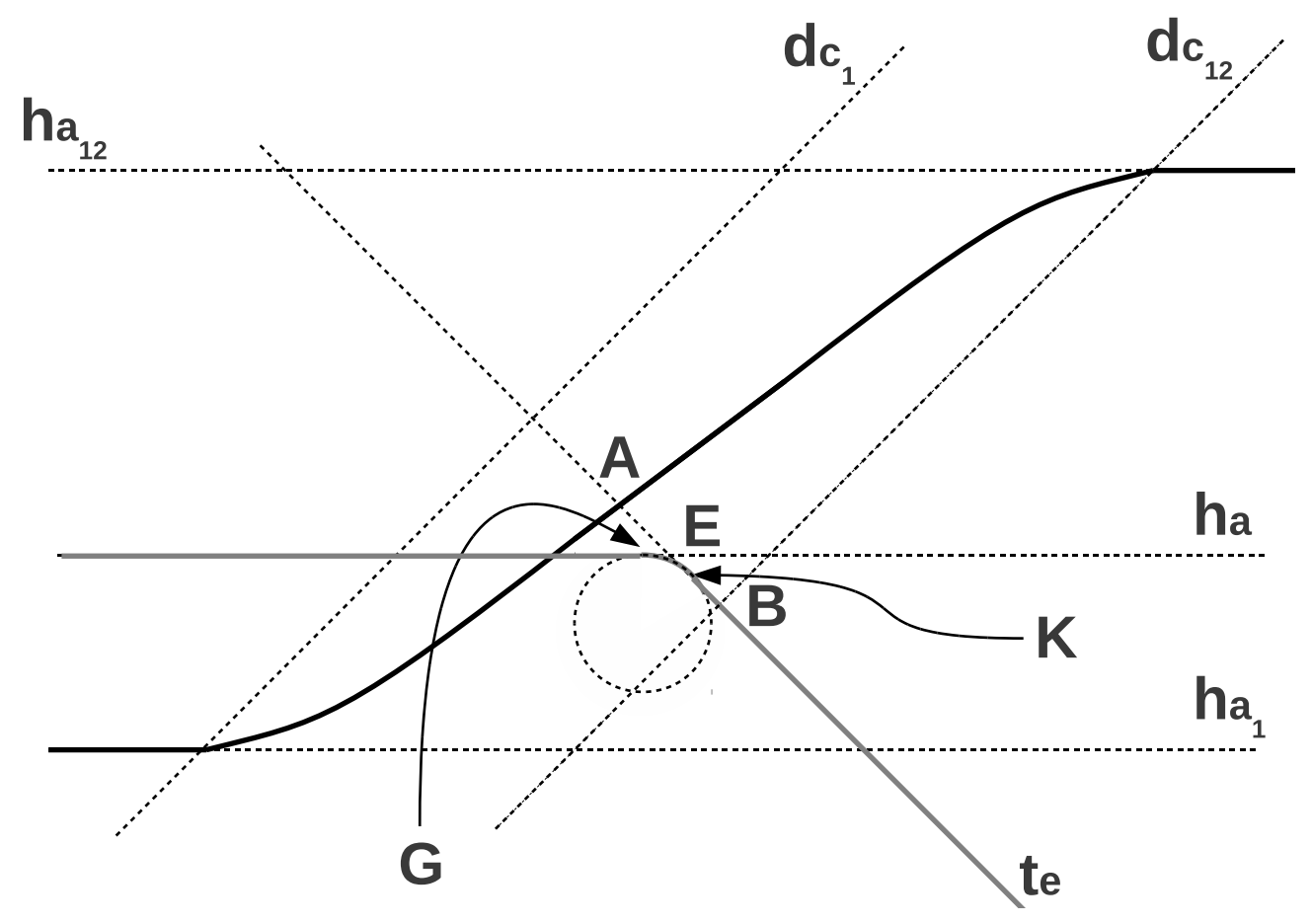

Fig. 2

We notice that the facets of $\Gamma$, near each vertex of $\Gamma$, are represented with the help of piecewise linear $v$. This fact is very important for our analysis.

Theorem 3.2. Let us suppose that $\Gamma(t)_{t \in[0, T)}$ is a family of gently bent rectangles, which are graphs of functions $v(\cdot, t)$ over a common reference manifold $\mathcal{M}$ (with the arc length parameter set $[0,2 \pi L)$ ) i.e. each $\Gamma(t)$ satisfies (3.3) for $t \in[0, T)$. Then,

(a) for each $t \in[0, T)$, the operator

$$
\frac{1}{\beta}\left(\kappa_{\gamma}+\sigma\right)
$$

on $\Gamma(t)$ takes the following form in variables $(s, t)$, at points where the normal to $\Gamma(t)$ is welldefined,

$$
\frac{a\left(v_{s}, v, s\right)}{\sqrt{(1+\kappa v)^{2}+v_{s}^{2}}}\left(\lambda\left(v_{s}, s\right)_{s}+\sigma\right) .
$$

Here, $\lambda(p, s)$ is given in (3.25) while a is defined by (3.31). Coefficient a is a positive, bounded Lipschitz continuous function, which is separated from zero. 
(b) Let us set

$$
W(p, s)=\varphi_{1}(s) \gamma_{\Lambda}|p|+\varphi_{2}(s)\left(\gamma_{\Lambda}|p-1|+\gamma_{R}|p+1|\right)+\varphi_{3}(s) \gamma_{R}|p|,
$$

where $\varphi_{i}(s):[0,2 \pi L) \rightarrow[0,1], i=1,2,3$, are chosen so that $\sum_{i=1}^{3} \varphi_{i}=1$ and

$$
\begin{aligned}
& \varphi_{1} \equiv 1 \text { for } s \in\left[0, s_{1}\right], \quad \varphi_{1} \equiv 0 \text { for } s \in\left[s_{2}, \frac{\pi}{2} L\right], \\
& \varphi_{2} \equiv 1 \text { for } s \in\left[s_{2}, s_{3}\right], \quad \varphi_{2} \equiv 0 \text { for } s \in\left[0, s_{1}\right] \cup\left[s_{4}, \frac{\pi}{2} L\right] \text {, } \\
& \varphi_{3} \equiv 1 \quad \text { for } s \in\left[s_{4}, \frac{\pi}{2} L\right), \quad \varphi_{3} \equiv 0 \text { for } s \in\left[0, s_{3}\right]
\end{aligned}
$$

and $s_{1}, s_{2}$ are given by (3.28) and (3.29), respectively. Points, $s_{3}$ and $s_{4}$ are defined in analogy to (3.28) and (3.29). Furthermore, this definition is extended by symmetry to $[0,2 \pi L)$.

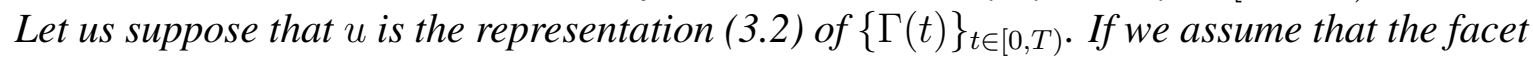
endpoints vary continuously in time, then (3.5) becomes

$$
\frac{a\left(v_{s}, v, s\right)}{\sqrt{(1+\kappa v)^{2}+v_{s}^{2}}}\left(\frac{\partial}{\partial s} W_{p}\left(v_{s}, s\right)+\tilde{\sigma}(v, s, t)\right)
$$

where $\tilde{\sigma}$ is defined by (3.32). If $\left\{\Gamma^{\prime}(t)\right\}_{t \in[0, T)}$ is another family of gently bent rectangles over $\mathcal{M}$ varying continuously with $t$ in the $C^{0}$ topology with $\Gamma(0)=\Gamma^{\prime}(0)$, then (3.5) takes the form (3.9).

(c) If $V$ is the velocity of $\Gamma$ in the normal direction, then

$$
V=\frac{v_{t}(1+\kappa v)}{\sqrt{v_{s}^{2}+(1+\kappa v)^{2}}},
$$

where $\kappa$ is the Euclidean curvature of $\mathcal{M}$.

Remarks. (1) An immediate consequence of this Theorem is that equation (1.1) takes the following form

$$
v_{t}=a\left(v_{s}, v, s\right)\left(W_{p}^{\prime}\left(v_{s}, s\right)_{s}+\tilde{\sigma}(v, s, t)\right)
$$

where $W$ (respectively, $a$ ) depends on $s$ (respectively, $s$ and $v$ ), while originally in [16] there is no such dependence. Thus, we may not directly apply the comparison principle of [16].

(2) If $(\Gamma, \xi)$ is a variational solution to (1.1), where $\Gamma(0)$ is a gently bent rectangle, with the corresponding $v(\cdot, t)$ appearing in (3.2), then we shall show that $v$ satisfies (3.11) in the viscosity sense. Obviously, not all solutions to (3.11) will yield bent rectangles by (3.2).

Before we engage in proving our statement we will present a time dependent partition of the arclength parameter set $[0,2 \pi L)$ of $\mathcal{M}$ corresponding to positions of facet endpoints. If we restrict first our attention to $\left[0, \frac{\pi}{2} L\right)$, then these points are:

$$
0<k_{\mathrm{I}}(t)<f_{1}<f_{2}^{l}<k_{\mathrm{II}}^{l}(t)<k_{\mathrm{II}}^{v}(t)<k_{\mathrm{II}}^{r}(t)<f_{2}^{r}<\frac{\pi}{2} L-k_{V}(t),
$$

where $k_{V}(t) \in\left(0, f_{3}\right)$. Since they are to correspond to facet endpoints, then by definition,

$$
\begin{array}{ll}
\Psi\left(k_{\mathrm{I}}(t), v\left(k_{\mathrm{I}}(t), t\right)\right)=\left(r_{0}(t), L_{0}(t)\right), & \Psi\left(k_{\mathrm{II}}^{l}(t), v\left(k_{\mathrm{II}}^{l}(t), t\right)\right)=\left(r_{1}(t), L_{1}(t)\right), \\
\Psi\left(k_{\mathrm{II}}^{v}(t), v\left(k_{\mathrm{II}}^{v}(t), t\right)\right)=\left(R_{1}(t), L_{1}(t)\right), & \Psi\left(k_{\mathrm{II}}^{r}(t), v\left(k_{\mathrm{II}}^{r}(t), t\right)\right)=\left(R_{1}(t), l_{1}(t)\right), \\
\Psi\left(\frac{\pi}{2} L-k_{V}(t), v\left(\frac{\pi}{2} L-k_{V}(t), t\right)\right)=\left(R_{0}(t), l_{0}(t)\right) . &
\end{array}
$$


This partition can be extended to the whole $[0,2 \pi L)$. For this purpose, we also introduce,

$$
\begin{aligned}
& U_{\mathrm{I}}^{0}=\left(-f_{1}, f_{1}\right), \quad U_{\mathrm{II}}^{0}=\left(f_{1}, f_{2}^{l}\right), \quad U_{\mathrm{II}}^{0}=\left(f_{2}^{l}, f_{2}^{r}\right), \\
& U_{\mathrm{IV}}^{0}=\left(f_{2}^{r}, \frac{\pi}{2} L-f_{3}\right), \quad U_{V}^{0}=\left(\frac{\pi}{2} L-f_{3}, \frac{\pi}{2} L+f_{3}\right) .
\end{aligned}
$$

Thus, we created a partition of $\mathcal{M}$ in the first quarter. Furthermore, we define

$$
U_{\mathrm{I}}^{1}=U_{\mathrm{I}}^{0}+\pi L, \quad U_{V}^{1}=U_{V}^{0}+\pi L .
$$

In order to describe other sets we need to introduce three mappings,

$$
\mathcal{Q}_{i}:\left[0, \frac{\pi}{2} L\right) \rightarrow\left[i \frac{\pi}{2} L,(i+1) \frac{\pi}{2} L\right), \quad i=1,2,3,
$$

by formulas

$$
\mathcal{Q}_{1}(s)=\pi L-s, \quad \mathcal{Q}_{2}(s)=\pi L+s, \quad \mathcal{Q}_{3}(s)=2 \pi L-s .
$$

Then, we set,

$$
U_{k}^{i}=\mathcal{Q}_{i}\left(U_{k}^{0}\right), \quad i=1,2,3, \quad k=\mathbb{I}, \mathbf{I I}, \mathbf{I V}
$$

and

$$
U_{k}=\bigcup_{i} U_{k}^{i}, \quad k=\mathrm{I}, \ldots, \mathrm{V}
$$

Proof of Theorem 3.2. We have to determine $\mathbf{n}$, the outer normal to $\Gamma$ at time $t$. Since $s$ is the arc-length parameter on $\mathcal{M}$, then due to (3.2) and Frenet formulas, we have

$$
\begin{aligned}
\frac{d}{d s}(x, y) & =\frac{d}{d s} \Psi=\frac{\partial}{\partial s}(\Phi(s)+\nu(s) v(s, t))=\tau(s)+\nu_{s}(s) v(s, t)+\nu(s) v_{s}(s, t) \\
& =\tau(s)(1+\kappa v(s, t))+\nu(s) v_{s}(s, t) .
\end{aligned}
$$

This formula is valid because in our setting ( $\mathcal{M}$ is negatively oriented) we have $\nu_{s}=\kappa \tau$, where $\kappa$ is the Euclidean curvature of $\mathcal{M}$. We also keep in mind that this tangent vector is negatively oriented. Hence,

$$
\mathbf{n}=\frac{-v_{s} \tau+(1+\kappa v) \nu}{\sqrt{v_{s}^{2}+(1+\kappa v)^{2}}}
$$

is the outer normal to $\Gamma(t)$. Furthermore,

$$
\mathbf{t}=-\frac{\tau(s)(1+\kappa v(s, t))+\nu(s) v_{s}(s, t)}{\sqrt{v_{s}^{2}+(1+\kappa v)^{2}}}
$$

is the unit tangent to $\Gamma(t)$ corresponding to the positive orientation of $\mathcal{M}$.

We recall that if $\xi$ is a vector field on $S$, then by definition,

$$
\operatorname{div}_{S} \xi:=\mathbf{t} \cdot \frac{\partial \xi}{\partial \mathbf{t}}
$$

In order to find the form of (1.1) in the local coordinates, we apply the following procedure: (1) calculate $\nabla \gamma$; 
(2) calculate $\frac{\partial}{\partial \mathbf{t}} \nabla \gamma$, where $\mathbf{t}$ is the unit normal corresponding to the positive orientation of $\Gamma$;

(3) calculate $\mathbf{t} \cdot \frac{\partial}{\partial \mathbf{t}} \nabla \gamma$.

In fact we will use $s \mapsto \Psi(s, v(s, t), t)$ to parametrize $\Gamma(t)$, but we have to be aware that it is not any arclength parameter $s_{a}$ on $\Gamma(t)$. We notice that,

$$
\frac{\partial \xi}{\partial \mathbf{t}}=\frac{d \xi}{d s_{a}}=\frac{\partial \xi}{\partial s} \frac{d s}{d s_{a}}=\frac{\partial \xi}{\partial s} \frac{1}{\left|\frac{d \Psi}{d s}\right|} .
$$

Let us notice that, if $s \in\left(-k_{\mathrm{I}}(t), k_{\mathrm{I}}(t)\right.$, then $v_{s}=0$ and due to (3.19)

$$
\operatorname{div}_{S} \xi=-\frac{1}{\left|\frac{d \Psi}{d s}\right|} \frac{\partial \xi_{1}}{\partial s} .
$$

The minus corresponds to the fact that $s$ sets the negative orientation. For $s \in\left(-k_{\mathrm{I}}(t), k_{\mathrm{I}}(t)\right)$ it is true that $\left|\Psi_{s}\right|=1$ and the normal $\mathbf{n}$ to $\Gamma(t)$ is $(0,1)$. Thus, $\gamma$ takes the form, in a neighborhood of $\mathbf{n}$,

$$
\gamma\left(\tilde{p}_{1}, \tilde{p}_{2}\right)=\gamma_{\Lambda}\left|\tilde{p}_{1}\right|+\gamma_{R} \tilde{p}_{2}
$$

Hence,

$$
\partial \gamma\left(\tilde{p}_{1}, \tilde{p}_{2}\right)=\gamma_{\Lambda} \partial\left|\tilde{p}_{1}\right| \mathbf{e}_{1}+\gamma_{R} \mathbf{e}_{2},
$$

where $\mathbf{e}_{1}$ and $\mathbf{e}_{2}$ are the unit vectors of the axes.

Taking this into account, (3.21) and the definition of $\kappa_{\gamma}$ at least formally we arrive at

$$
\kappa_{\gamma}=\gamma_{\Lambda} \frac{\partial}{\partial s}\left(\operatorname{sgn} v_{s}\right), \quad s \in\left(-k_{\mathrm{I}}(t), k_{\mathrm{I}}(t)\right) .
$$

In other words,

$$
\lambda(p, s)=\gamma_{\Lambda} \operatorname{sgn} p \quad \text { for } s \in\left(-k_{\mathrm{I}}(t), k_{\mathrm{I}}(t)\right) .
$$

Of course, we will take advantage of the fact that $\operatorname{sgn}(p)$ is a multivalued function.

Exactly the same argument applies for $\left(\frac{\pi}{2} L-k_{V}(t), \frac{\pi}{2} L+k_{V}(t)\right)$, however there

$$
\gamma\left(\tilde{p}_{1}, \tilde{p}_{2}\right)=\gamma_{\Lambda} \tilde{p}_{1}+\gamma_{R}\left|\tilde{p}_{2}\right|,
$$

and as a result

$$
\partial \gamma\left(\tilde{p}_{1}, \tilde{p}_{2}\right)=\gamma_{\Lambda} \mathbf{e}_{1}+\gamma_{R} \partial\left|\tilde{p}_{2}\right| \mathbf{e}_{2}
$$

Hence,

$$
\lambda(p, s)=\gamma_{R} \operatorname{sgn}(p) \quad \text { for } s \in\left(\frac{\pi}{2} L-k_{V}(t), \frac{\pi}{2} L+k_{V}(t)\right) .
$$

Now we consider the interval $\left[k_{\mathrm{I}}(t), f_{1}\right]$ of the parametrization of $\Gamma(t)$. The normal vector is different from $(0,1)$, hence $\gamma\left(\tilde{p}_{1}, \tilde{p}_{2}\right)=-\gamma_{\Lambda} \tilde{p}_{1}+\gamma_{R} \tilde{p}_{2}$. We conclude that

$$
\nabla \gamma\left(\tilde{p}_{1}, \tilde{p}_{2}\right)=\left(-\gamma_{\Lambda}, \gamma_{R}\right)
$$

The result does not depend upon $\tilde{p}$, hence we take $\lambda(p, s)=$ const. For the sake of consistency with (3.23) we set

$$
\lambda(p, s)=\gamma_{\Lambda}
$$


Let us consider intervals $s \in\left(k_{\mathrm{I}}(t), k_{\mathrm{II}}^{l}(t)\right) \cup\left(k_{\mathrm{II}}^{r}(t), \frac{\pi}{2} L-k_{V}(t)\right)$. In this case the normal to $\Gamma(t)$ at $\Psi(s, v(s), t)$ is different from $(0,1)$ and $(1,0)$, hence $\gamma\left(\tilde{p}_{1}, \tilde{p}_{2}\right)=-\gamma_{\Lambda} \tilde{p}_{1}+\gamma_{R} \tilde{p}_{2}$. Thus,

$$
\nabla \gamma\left(\tilde{p}_{1}, \tilde{p}_{2}\right)=\left(-\gamma_{\Lambda}, \gamma_{R}\right)
$$

In other words, $\xi=\left(-\gamma_{\Lambda}, \gamma_{R}\right)$, so $\frac{\partial \xi}{\partial \mathbf{t}}=0$, hence $\kappa_{\gamma}=0$. Performing calculation similar to that above, we conclude that for $s \in\left(k_{\mathrm{I}}(t), k_{\mathrm{II}}^{l}(t)\right) \cup\left(k_{\mathrm{II}}^{r}(t), \frac{\pi}{2} L-k_{V}(t)\right)$ function $\lambda(p, s)$ depends only on $s$. Its exact value is given in (3.25).

Let us consider interval $\left(k_{\mathrm{II}}^{l}(t), k_{\mathrm{II}}^{r}(t)\right)$. For $s \in\left(k_{\mathrm{II}}^{l}(t), k_{\mathrm{II}}^{v}(t)\right)$, the normal vector to $\Gamma(t)$ at $\Psi(s, v(s, t))$ is $(0,1)$, while for $s \in\left(k_{\mathbb{I I}}^{v}(t), k_{\mathbb{I I}}^{r}(t)\right)$ the normal vector is $(1,0)$.

Taking into account the form of the normal vector at $\Psi(s, v(s, t))$ for $s \in\left(k_{\mathrm{II}}^{l}(t), k_{\mathrm{II}}^{v}(t)\right)$, we have to compute

$$
\nabla \gamma\left(\tilde{p}_{1}, \tilde{p}_{2}\right)=\nabla\left(\gamma_{\Lambda}\left|\tilde{p}_{1}\right|+\gamma_{R} \tilde{p}_{2}\right) .
$$

We observe that $\left|\Psi_{s}\right|=\sqrt{2}$ for $s \in\left(k_{\mathbb{I I}}^{l}(t), k_{\mathbb{I I}}^{r}(t)\right)$. The normal to the graph of $v$ for $s \in$ $\left(k_{\mathrm{II}}^{l}(t), k_{\mathrm{II}}^{v}(t)\right)$ is

$$
\mathbf{n}=\frac{1}{\sqrt{v_{s}^{2}+1}}\left(-1, v_{s}\right)
$$

In order to proceed, we have to express the variables $\left(\tilde{p}_{1}, \tilde{p}_{2}\right)$ used to compute $\nabla \gamma$ in the local coordinate system, $\left(p_{1}, p_{2}\right)$ related to $\mathcal{M}$. We notice that

$$
\tilde{p}_{1}=\frac{p_{1}+p_{2}}{\sqrt{2}}, \quad \tilde{p}_{2}=\frac{p_{2}-p_{1}}{\sqrt{2}} .
$$

Hence, after taking into account that $\left|\Psi_{s}\right|=\sqrt{2}$ for $s \in\left(k_{\mathrm{II}}^{l}(t), k_{\mathrm{II}}^{v}(t)\right)$, we obtain

$$
\lambda(p, s)=\frac{1}{\sqrt{2}} \gamma_{\Lambda} \operatorname{sgn}(p-1) .
$$

We stress that $k_{\mathbb{I I}}^{v}(t)$, the point separating $\left(k_{\mathbb{I I}}^{l}(t), k_{\mathbb{I I}}^{r}(t)\right)$ into two pieces, where $v_{s}= \pm 1$ depends upon time.

A similar calculation for $s \in\left(k_{\mathrm{II}}^{v}(t), k_{\mathrm{II}}^{r}(t)\right)$ lead us to the conclusion that

$$
\lambda(p, s)=\frac{1}{\sqrt{2}} \gamma_{\Lambda} \operatorname{sgn}(p+1) .
$$

The argument presented above shows that in the local coordinates which we use here, the singular directions are,

$$
p=+1 \quad \text { on }\left(k_{\mathbb{I I}}^{l}(t), k_{\mathbb{I I}}^{v}(t)\right) \text { and } p=-1 \quad \text { on }\left(k_{\mathbb{I I}}^{v}(t), k_{\mathbb{I I}}^{r}(t)\right) .
$$

What is left is region $U_{\mathrm{IV}}$, there the calculation are as for region $U_{\mathbb{I I}}$.

The results obtained so far may be summarized in the following way,

$$
\lambda(p, s)= \begin{cases}\gamma_{\Lambda} \operatorname{sgn} p & s \in\left(-k_{I}(t), k_{I}(t)\right), \\ \gamma_{\Lambda} /\left|\frac{d \Psi}{d s}\right| & s \in\left(k_{I}(t), k_{\mathrm{II}}^{l}(t)\right), \\ \frac{1}{\sqrt{2}} \gamma_{\Lambda} \operatorname{sgn}(p-1) & s \in\left(k_{\mathrm{II}}^{l}(t), k_{\mathrm{II}}^{v}(t)\right), \\ \frac{1}{\sqrt{2}} \gamma_{R} \operatorname{sgn}(1+p) & s \in\left(k_{\mathrm{II}}^{v}(t), k_{\mathrm{II}}^{r}(t)\right), \\ \gamma_{R} /\left|\frac{d \Psi}{d s}\right| & s \in\left(k_{\mathrm{II}}^{r}(t), \frac{\pi}{2} L-k_{V}(t)\right), \\ \gamma_{R} \operatorname{sgn} p & s \in\left(\frac{\pi}{2} L-k_{V}(t), \frac{\pi}{2} L+k_{V}(t)\right),\end{cases}
$$


where we made a choice of $\lambda(p, s)$ when ambiguity arose.

Of course, we can extend it by symmetry to the whole $[0,2 \pi L)$.

This provides the main result of part (a). At this moment it is convenient to go straight to the form of $W$ in part (b).

It is also clear from the structure of the equations involving $W$ that $W$ is determined up to an affine function. We also see that $k_{\mathrm{II}}^{v}(t)$ looks like a free boundary. In fact it is not, because it is the projection of the intersection of the lines containing the facets meeting at the vertex. That is, it is sufficient to know these lines.

At this point, it is evident from (3.25) that even if we succeed in writing (3.5) as

$$
v_{t}=a \cdot\left(\frac{\partial}{\partial s} W_{p}\left(v_{s}\right)+\sigma\right)
$$

then $W=W(p, s)$, i.e., $W$ will explicitly depend upon $s$.

We first take care of the apparent free boundary $k_{\mathbb{I I}}^{v}(t)$. We notice that if we choose $W$ as follows,

$$
W(p, s)=\gamma_{\Lambda}|p-1|+\gamma_{R}|1+p|
$$

for $s \in\left(k_{\mathbb{I I}}^{l}(t), k_{\mathbb{I I}}^{r}(t)\right)$, then the equation we study takes the desired form (3.26) and point $k_{\mathrm{II}}^{v}(t)$ is no longer explicitly mentioned. Moreover,

$$
\frac{\partial}{\partial s}\left(\frac{\partial W}{\partial p}\left(v_{s}, s\right)\right)=\frac{\partial}{\partial s} \lambda\left(v_{s}, s\right)
$$

Indeed, this is so, because at $p_{0}=1$ we have

$$
\frac{\partial W}{\partial p}=\frac{\partial}{\partial p}\left(\gamma_{\Lambda}|p-1|+\gamma_{R}|1+p|\right)=\gamma_{\Lambda} \operatorname{sgn} p_{0}+\gamma_{R}
$$

so (3.27) follows.

It is also obvious from the form of $\lambda(p, s)$, see (3.25), that we may write

$$
W(p, s)= \begin{cases}\gamma_{\Lambda}|p|, & s \in\left(-k_{\mathrm{I}}(t), k_{\mathrm{I}}(t)\right) \\ \gamma_{R}|p|, & s \in\left(\frac{\pi}{2} L-k_{V}(t), \frac{\pi}{2} L+k_{V}(t)\right)\end{cases}
$$

We would rather expect that $W$ is sufficiently regular, as well as $a$ and $\sigma$. The present formula for $W$ requires a smooth transition between the different forms. We will suggest a new one so that we will not introduce spurious singular directions at a given solution. This remark matters, because interval $\left[f_{1}, k_{\mathrm{II}}^{l}(t)\right)$ corresponding to the curved part of $\Gamma$ contains a set, where $v_{s}(0, s)>1$. This is obvious, since for $s$ close to $k_{\mathrm{II}}^{l}(0)$, we have $v_{s}(0, s)>1$. Due to the continuity of $v_{s}$, there are $t_{1}$ and $s_{1} \in\left[f_{1}, k_{\mathbb{I I}}^{l}(0)\right)$ such that we have

$$
v_{s}(t, s)>1 \text { for } s>s_{1}, t \in\left(0, t_{1}\right) .
$$

We take again $k_{\text {III }}^{l}$ defined in (3.12) and we set $s_{2}$ by

$$
s_{2}=\frac{s_{1}+k_{\mathrm{II}}^{l}}{2}
$$


The same analysis applies to $\left(k_{\mathbb{I I}}^{r}(t), f_{2}^{r}\right]$ yielding $s_{3}, s_{4} \in\left(k_{\mathbb{I I}}^{r}(t), f_{2}^{r}\right]$ and the same $t_{1}$, where $v_{s}(t, s)<-1$.

Thus, we set

$$
W(p, s)= \begin{cases}\gamma_{\Lambda}|p| & \text { for } s \in\left[0, s_{1}\right) \\ \gamma_{\Lambda}|p| \varphi_{1}(s)+\frac{1}{\sqrt{2}}\left(\gamma_{\Lambda}|p-1|+\gamma_{R}|p+1|\right)\left(1-\varphi_{1}(s)\right) & \text { for } s \in\left(s_{1}, s_{2}\right) \\ \gamma_{\Lambda}|p-1|+\gamma_{R}|p+1| & \text { for } s \in\left(s_{2}, k_{\mathbb{I I}}^{r}\right)\end{cases}
$$

where $\varphi \in C_{0}^{\infty}(\mathbb{R})$, such that $\varphi \in[0,1], \varphi \equiv 0$ for $s \leq s_{1}$ and $\varphi \equiv 1$ for $s \geq s_{2}$.

We can perform a similar type of analysis for the endpoint $\left(R_{1}, l_{1}\right)$. Summing up, we arrive at formulae (3.7), (3.8).

This definition of $W$ requires a change in $\sigma$, because for $s \in\left(s_{1}, s_{2}\right)$ we have (similar situation occurs on $\left(s_{3}, s_{4}\right)$ ),

$$
\begin{aligned}
\frac{\partial}{\partial s} W_{p}\left(v_{s}, s\right) & =\frac{\partial}{\partial s}\left(\gamma_{\Lambda} \varphi_{1} \operatorname{sgn} v_{s}+\frac{1}{\sqrt{2}}\left(\gamma_{\Lambda} \operatorname{sgn}\left(v_{s}-1\right)+\gamma_{R} \operatorname{sgn}\left(v_{s}+1\right)\right)\left(1-\varphi_{1}\right)\right) \\
& =\left(\gamma_{\Lambda}\left(1-\frac{1}{\sqrt{2}}\right)-\gamma_{R} \frac{1}{\sqrt{2}}\right) \varphi_{1, s}(s) .
\end{aligned}
$$

Thus, we will have to adjust the definition of $\tilde{\sigma}$.

Now, we turn our attention to $a=1 / \beta(\mathbf{n})$. After recalling the formula (3.17) for $\mathbf{n}(s)$, valid for all $s \in \mathbb{T}^{1}$, we come to the conclusion that

$$
\beta(\mathbf{n}(s))=\beta\left(\frac{\left.(1+\kappa(s) v(s, t)) \nu(s)-v_{s}(s, t) \tau(s, t)\right)}{\sqrt{(1+\kappa(s) v(s, t))^{2}+v_{s}^{2}(s, t)}}\right) .
$$

Hence, we set

$$
a(p, v, s)=\sqrt{(1+\kappa v)^{2}+p^{2}} / \beta\left(\frac{(1+\kappa v) \nu-p \tau}{\sqrt{(1+\kappa v)^{2}+p^{2}}}\right) .
$$

We note that this definition of $a$ includes the factor appearing in the denominator of formula (3.10), where $v_{s}$ is replaced by $p(3.18)$.

We notice that, function $p \rightarrow a(p, v, s)$ is Lipschitz once we assume such a condition on $\beta$. In addition, $\beta$ is one-homogeneous, vanishing at zero. Since it is evaluated at a normal vector, then in all regions from $U_{I}$ to $U_{V}$ we will have

$$
a_{0} \leq a \leq a_{1}
$$

Equation (3.5) contains also the term $\sigma=\sigma\left(x_{1}, x_{2}, t\right)$. Since for a given $t>0$, we have that

$$
\left(x_{1}, x_{2}\right)=\Psi(s, v(s, t)) .
$$

In other words,

$$
\tilde{\sigma}(v, s, t)=\sigma(\nu(s) v+\Phi(s))+\left(\gamma_{R} \frac{2-\sqrt{2}}{2}-\gamma_{\Lambda} \frac{1}{\sqrt{2}}\right) \varphi_{3, s}(s)-\left(\gamma_{\Lambda} \frac{2-\sqrt{2}}{2}-\gamma_{R} \frac{1}{\sqrt{2}}\right) \varphi_{1, s}(s) .
$$


The properties of $\tilde{\sigma}$ will be discussed below.

(c) Let us notice that

$$
\frac{d}{d t}\left(x_{1}, x_{2}\right)=\frac{d}{d t}(\Phi(s)+\nu(s) v(s, t))=\nu(s) v_{t}(s, t) .
$$

Since $V=\frac{d}{d t}\left(x_{1}, x_{2}\right) \cdot \mathbf{n}$, where $\mathbf{n}$ is the outer normal to $\Gamma$, then taking into account (3.17) yields the desired formula (3.10).

We will see that $\tilde{\sigma}$ inherits properties of $\sigma$ implied by Berg's effect. The most important for us is its dependence on $v$.

Lemma 3.1. Let us suppose that $\sigma$ satisfies Bergs' effect. If $\tilde{\sigma}$ is defined by (3.32), then the map $v \longmapsto \tilde{\sigma}(v, s, t)$ is monotone.

Proof. Let us write $\nu(s)=\left(\nu_{1}(s), \nu_{2}(s)\right)$. Then

$$
\frac{\partial}{\partial v} \tilde{\sigma}=\nu_{1} \frac{\partial}{\partial e_{1}} \sigma+\nu_{2} \frac{\partial}{\partial e_{2}} \sigma
$$

Due to (2.1), we have $\nu_{1} \frac{\partial}{\partial e_{1}} \sigma$ and $\nu_{2} \frac{\partial}{\partial e_{2}} \sigma$ are positive, hence $\frac{\partial}{\partial v} \tilde{\sigma}>0$.

\section{When variational solutions are viscosity solutions?}

We want to show that the variational solutions we constructed in [18] are indeed viscosity solutions in the sense of [16]. For this purpose, we recall the definition of viscosity sub/supersolution with a simplifying continuity assumption. We note our equation (3.11) differs from the topic of the study in [16]. Potentially, this is a very serious problem, but in our case we circumvent the difficulties.

It should be stressed that in our construction of variational solutions in [18], we assumed that the verteces $\left( \pm R_{1}, \pm L_{1}\right)$ move as intersections of the lines containing the outer facets. In general, this need not be the case. So we establish here a sufficient condition for this property to hold.

\subsection{Viscosity solutions}

In [16], we developed a framework of viscosity solutions to problems of the form

$$
v_{t}=a\left(v_{x}\right)\left(\frac{\partial}{\partial x} W_{p}\left(v_{x}\right)+\sigma(x, t)\right)
$$

with periodic boundary conditions, where $W$ is merely a convex, but not a $C^{1}$ function. One of the problems there was to give meaning to $\frac{\partial}{\partial x} W_{p}\left(v_{x}\right)$. This is done by solving a minimization problem on an interval. Here, functions $a$ and $W$ depend on additional variables, however, when restricted to facets of $\Gamma$ this additional dependence disappears. This permits us to use the definition of the viscosity solutions developed in [16]. In order to avoid unnecessary technicalities, we shall present the definitions for continuous super-/subsolutions. 
We would like to define viscosity solutions for problem (3.11), i.e.,

$$
v_{t}=a\left(v_{x}, v, x\right)\left(W_{p}^{\prime}\left(v_{x}, x\right)_{x}+\tilde{\sigma}(v, x, t)\right),
$$

with periodic boundary conditions. We assume that $p \mapsto W(p, x)$ is convex, but not necessarily $C^{1}$. For each $x \in \mathbb{T}^{1}$, we have a finite set

$$
\mathcal{P}(x)=\left\{p_{1}(x), \ldots, p(x)_{k(x)}\right\},
$$

of points of nondifferentiability of $W(\cdot, x)$. We shall also assume that

$$
x \mapsto W(p, x) \text { is a smooth function, }
$$

$a$ is Lipschitz continuous, $\sigma \in C^{1}\left(\mathbb{R}^{2} \times \mathbb{R}_{+}\right)$and there is $\bar{M}>0$ such that

$$
\frac{\partial}{\partial u}(a \sigma) \leq \bar{M}
$$

In $[16, \S 2]$, we gave a variational characterization of the quantity $\Lambda_{W}^{\sigma}$, which is formally defined by

$$
\Lambda_{W}^{\sigma}(u)(x)=\left(W^{\prime}\left(u_{x}\right)\right)_{x}+\sigma(x) .
$$

Here, we have to extend it to include the dependence of $\sigma$ upon $u$ and we have to explain how to proceed, when $W$ depends on $x$. However, the last question is easiest, because $W$ restricted to faceted regions of $u$ is independent of $x$.

For the purpose of the definition, we assume that $Z$ is a real-valued $C^{2}$-function, defined in a bounded interval $\bar{I}$, where $I=(a, b) \subset \mathbb{T}^{1}$. For a given $\Delta>0$, we define $K_{\chi_{l} \chi_{r}}^{Z}$ to be the set of all $\xi \in H^{1}(I)$, satisfying

$$
Z(x)-\Delta / 2 \leq \xi(x) \leq Z(x)+\Delta / 2 \text { for } x \in I \text { (the obstacle condition) }
$$

and

$$
\xi(a)=Z(a)-\chi_{l} \Delta / 2, \xi(b)=Z(b)+\chi_{r} \Delta / 2 \text { (boundary conditions). }
$$

Here, $\chi_{l}$ and $\chi_{r}$ take values \pm 1 and they will be defined in (4.8), (4.9).

Let $J_{\chi_{l} \chi_{r}}^{Z}$ be the functional on $L^{2}(I)$, defined by

$$
J_{\chi_{l} \chi_{r}}^{Z}(\xi)= \begin{cases}\int_{a}^{b}\left|\xi^{\prime}(x)\right|^{2} d x, & \xi \in K_{\chi_{l} \chi_{r}}^{Z} \\ \infty, & \text { otherwise. }\end{cases}
$$

For $\xi \in H^{1}(I)$, we define the coincidence set $D_{ \pm}(\xi)$ by

$$
D_{ \pm}=D_{ \pm}(\xi)=\{x \in \bar{I} \mid \xi(x)=Z(x) \pm \Delta / 2\} .
$$

We say that $D_{+}$is the upper coincidence set while $D_{-}$is the lower coincidence set.

Definition 4.1. We say that $\xi \in K_{\chi_{l} \chi_{r}}$ satisfies the concave-convex condition if $\xi$ is concave on each connected component of the complement of the upper coincidence set $D_{+}$and convex on each connected component of the complement of the lower coincidence set $D_{-}$, i.e., $\xi^{\prime \prime} \leq 0$ outside $D_{+}$and $\xi^{\prime \prime} \geq 0$ outside $D_{-}$. In particular, $\xi^{\prime \prime}=0$ outside $D_{-} \cup D_{+}$. 
Proposition 4.1. ([16, A characterization of the minimizer, Proposition 1]) Function $\xi_{\chi_{l} \chi_{r}} \in$ $K_{\chi_{l} \chi_{r}}$ is the minimizer of $J_{\chi_{l} \chi_{r}}$ if and only if $\xi_{\chi_{l} \chi_{r}}$ fulfills the concave-convex condition. In particular, $\xi_{\chi_{l} \chi_{r}}$ is $C^{1,1}$ in $I=(a, b)$ and

$$
\sup _{x \in I}\left|\xi_{\chi_{l} \chi_{r}}^{\prime \prime}(x)\right| \leq \sup _{x \in I}\left|Z^{\prime \prime}(x)\right| .
$$

We set

$$
\Lambda_{\chi_{l} \chi_{r}}^{Z^{\prime}}(x, I)=\frac{d \xi_{\chi_{l} \chi_{r}}(x)}{d x}
$$

Of course, $\xi_{\chi_{l} \chi_{r}}$ depends also on interval $I$, but we suppress this in the notation.

If $W$ depends only on $p$ and we fix $p_{0} \in \mathcal{P}, I=(a, b), \sigma=\sigma(x, t)$, then it is easy to observe that $\Lambda_{\chi_{l} \chi_{r}}^{Z^{\prime}}$ agrees with $\eta_{x}^{0}+\sigma$, when $Z$ is a primitive of $\sigma$ and $\eta^{0}$ minimizes

$$
\int_{I}\left|\eta_{x}+\sigma\right|^{2}
$$

over

$$
\left\{\eta \in H^{1}(I): \eta(x) \in \partial W\left(p_{0}\right), \eta(a)=-\chi_{l} \frac{\Delta}{2}, \eta(b)=\chi_{r} \frac{\Delta}{2}\right\}
$$

It is sufficient to take $\xi=\eta+Z$. The reason we write $Z^{\prime}$ instead of $Z$ is that the derivative of $\xi_{\chi_{l} \chi_{r}}^{Z}$ depends on $Z$ only through its derivative. Once we fix $Z$, we suppress $Z^{\prime}$ in (4.7). We shall write $\Lambda_{-+}$etc. instead of writing $\Lambda_{\{-1\},\{+1\}}$.

We recall an important result needed further in this paper.

Theorem 4.1. ([16, Comparison principle, Theorem 1]) Assume that $I_{1}$ and $I_{2}$ are bounded open intervals.

(i) If $I_{2} \subset I_{1}$, then

$$
\Lambda_{--}\left(x, I_{2}\right) \leq \Lambda_{ \pm \pm}\left(x, I_{1}\right) \leq \Lambda_{++}\left(x, I_{2}\right) \text { for } x \in I_{2} .
$$

(ii) If $a \leq c<b \leq d$ for $I_{1}=(a, b), I_{2}=(c, d)$, then for $x \in(c, b)$

$$
\Lambda_{ \pm-}\left(x, I_{1}\right) \leq \Lambda_{+ \pm}\left(x, I_{2}\right), \Lambda_{- \pm}\left(x, I_{2}\right) \leq \Lambda_{ \pm+}\left(x, I_{1}\right) .
$$

We recall a notion of a faceted function. Let $\Omega$ be an open interval. A function $f \in C(\Omega)$ is called faceted at $x_{0}$ with slope $p$ on $\Omega$ (or p-faceted at $x_{0}$ ) if there is a closed nontrivial finite interval $I(\subset \Omega)$, containing $x_{0}$ such that $f$ agrees with an affine function

$$
\ell_{p}(x)=p\left(x-x_{0}\right)+f\left(x_{0}\right) \text { in } I
$$

and $f(x) \neq \ell_{p}(x)$ for all $x \in J \backslash I$ with a neighborhood $J(\subset \Omega)$ of $I$. Interval $I$ is called the faceted region of $f$ containing $x_{0}$ and is denoted by $R\left(f, x_{0}\right)$. The set of continuous $p$-faceted functions on $\Omega$ and with $p \in \mathcal{P}$ will be called $C_{P}(\Omega)$.

Since our set $\mathcal{P}=\mathcal{P}(x)$ depends upon the space variable, we have to modify the original definition of the $\mathcal{P}$-faceted function. We will use the fact that for all $x \in U_{j}$ we have $\mathcal{P}(x) \equiv P_{j}$, where

$$
P_{\mathrm{I}}=P_{\mathrm{V}}=\{0\}, \quad P_{\mathrm{III}}=\{-1,1\}, \quad P_{\mathrm{II}}=P_{\mathrm{IV}}=\{-1,0,1\} .
$$


However, the solutions will avoid the singular slopes for $x \in P_{\mathrm{II}} \cup P_{\mathrm{IV}}$.

We will say that $f$, a continuous function on $\Omega \subset \mathbb{T}^{1}$ or a function from $C_{p e r}(0,2 \pi L)$, is $\mathcal{P}_{j}$-faceted function at $x_{0} \in P_{\mathrm{I}} \cup P_{\mathrm{II}} \cup P_{\mathrm{V}}$, if $f$ restricted to a component $U_{k}^{i}$ of this set containing $x_{0}$ is $p$-faceted with $p$ in $\mathcal{P}_{k}$.

We introduce the left transition number $\chi_{l}=\chi_{l}\left(f, x_{0}\right)$ and the right transition number $\chi_{r}=\chi_{r}\left(f, x_{0}\right)$ by

$$
\begin{gathered}
\chi_{l}=\left\{\begin{array}{lll}
+1 \text { if } f \geq \ell_{p_{i}} \text { in } & \left\{x \in J \mid x \leq x_{0}\right\}, \\
-1 \text { if } f \leq \ell_{p_{i}} \text { in } & \left\{x \in J \mid x \leq x_{0}\right\},
\end{array}\right. \\
\chi_{r}=\left\{\begin{array}{lll}
+1 \text { if } f \geq \ell_{p_{i}} \text { in } & \left\{x \in J \mid x \geq x_{0}\right\}, \\
-1 \text { if } f \leq \ell_{p_{i}} \text { in } \quad\left\{x \in J \mid x \geq x_{0}\right\},
\end{array}\right.
\end{gathered}
$$

if $f$ is $p_{i}$-faceted at $x_{0}$.

Definition 4.2. (see [16, Definition 2]) We assume that $S$ is a real-valued Lipschitz function on an open interval $\Omega=(a, b)$ and $Z$ is its primitive. Moreover, $W$ is given by (3.7) and $\Omega$ is contained in one of the connected components of $U_{I} \cup U_{\text {III }} \cup U_{V}$. We assume that $f \in C(\Omega)$ $p_{i}$-faceted at $x_{0} \in \Omega$ with $p_{i} \in P\left(x_{0}\right)$. Then, we define the nonlocal curvature $\Lambda_{W}^{S}$ by

$$
\Lambda_{W}^{S}(f)\left(x_{0}\right):=\Lambda_{\chi_{l} \chi_{r}}^{Z^{\prime}}\left(x_{0}, I\right)
$$

the right hand side is defined by (4.7) with $\Delta=W^{\prime}\left(p_{i}+0, x_{0}\right)-W^{\prime}\left(p_{i}-0, x_{0}\right)$ and $I$ is the faceted region $R\left(f, x_{0}\right)$. If $f$ is twice differentiable at $x_{0}$ and $f^{\prime}\left(x_{0}\right) \notin P\left(x_{0}\right)$, we set, as expected,

$$
\Lambda_{W}^{\sigma}(f)\left(x_{0}\right)=W^{\prime \prime}\left(f^{\prime}\left(x_{0}\right)\right) f^{\prime \prime}\left(x_{0}\right)+\sigma\left(x_{0}\right) .
$$

However, we have to address the situation, when $\tilde{\sigma}$ in (4.1) depends on $u$ and possibly on $u_{x}$. For this purpose, we adjust Definition 4.2. Let us suppose that $S: \mathbb{R}^{3} \rightarrow \mathbb{R}$ is Lipschitz continuous, $f \in C_{P}^{2}(\Omega)$ is $p$-faceted at $x_{0} \in \Omega, p \in \mathcal{P}\left(x_{0}\right)$ and $\Omega \cap\left(U_{\mathbb{I}} \cup U_{\mathrm{IV}}\right)=\emptyset, g \in C(\bar{\Omega})$. We define $\Lambda_{W}^{S}(f, g)$ by the following formula,

$$
\Lambda_{W}^{S}(f, g)\left(x_{0}\right):=\Lambda_{\chi_{l} \chi_{r}}^{Z^{\prime}}\left(x_{0}, I\right)
$$

where $I=R\left(f, x_{0}\right)$ is the faceted region of $f$ and we set

$$
Z(x)=C+\int_{0}^{x} S\left(f^{\prime}(s), g(s), s\right) d s .
$$

As before, we set $\Delta=W_{p}\left(p+0, x_{0}\right)-W_{p}\left(p-0, x_{0}\right)$. If $f$ is twice differentiable at $x_{0}$ and $f^{\prime}\left(x_{0}\right) \notin \mathcal{P}\left(x_{0}\right)$, then

$$
\Lambda_{W}^{S}(f, g)\left(x_{0}\right):=W_{p p}\left(f^{\prime}\left(x_{0}\right)\right) f^{\prime \prime}\left(x_{0}\right)+S\left(f^{\prime}\left(x_{0}\right), g\left(x_{0}\right), x_{0}\right) .
$$

We would like to state a simple observation, which will be very useful later. But first we state a useful definition.

Since we consider facets only over parts of the reference manifold, $\mathcal{M}$ we introduce a new notion reflecting that. 
Definition 4.3. We say that a continuous, real valued function $h$ defined on $\mathbb{T}^{1}$ is a profile function of a gently bent rectangle at time $t$ if:

(i) $h$ is faceted in $U_{I}^{0}$ (respectively, in $U_{V}^{0}$ ) with the slope equal to zero and it is monotone outside of the facet. Its transition numbers are +1 , so that near the facet $h$ is convex.

(ii) $h$ is strictly increasing in $U_{I I}^{0}$ (respectively, decreasing in $U_{N}^{0}$ ).

(iii) $h$ is faceted in $U_{\text {III }}^{0}$ with slope +1 and -1 which touches each other. Outside the facets $h$ is monotone. The transition numbers of both facets are -1 .

(iv) All upper and lower derivatives of houtside facets are away from zero.

(v) In $U_{\text {III }}^{0}$, the absolute values of upper and lower derivatives of $h$ outside facets are bigger than one.

(vi) In $U_{I}^{0}$ (respectively, in $U_{N}^{0}$ ) upper and lower derivatives of h are away from the singularity of $W(p, x)$.

(vii) The symmetric properties hold for the other components of $U_{\text {II }}$ (respectively, $U_{\text {III }}, U_{N}$ ).

(viii) On the facets of $h$, the quantity $\Lambda^{\tilde{\sigma}}(f, f)$ is constant (so that the facets do not break). This concept, however, depends on time.

Lemma 4.1. Let us suppose that $S: \mathbb{R}^{3} \rightarrow \mathbb{R}$ is Lipschitz continuous and for all $p, x$ the function $g \mapsto S(p, g, x)$ is decreasing. We assume that $f \in C_{P}^{2}$ is p-faceted at $x_{0}$ and $g_{1}, g_{2}$ are Lipschitz continuous profile functions and such that $g_{1} \leq g_{2}$. If additionally $\Lambda_{W}^{S}\left(f, g_{1}\right)$ is constant over $R\left(f, x_{0}\right)$, then

$$
\Lambda_{W}^{S}\left(f, g_{1}\right) \geq \Lambda_{W}^{S}\left(f, g_{2}\right)
$$

Proof. We set

$$
Z_{i}(x)=C_{i}+\int_{0}^{x} S\left(f^{\prime}(s), g_{i}(s), s\right) d s, \quad i=1,2 .
$$

and we assume that $R\left(f, x_{0}\right)=(a, b)$. We also select $C_{1}, C_{2}$ so that $Z_{1}(a)=Z_{2}(a)$. The solutions to the obstacle problem have to satisfy the boundary conditions

$$
\xi_{i}(a)=Z_{i}(a)-\chi_{l} \frac{1}{2} \Delta, \quad \xi_{i}(b)=Z_{i}(b)+\chi_{r} \frac{1}{2} \Delta, \quad i=1,2 .
$$

We assumed that $\Lambda_{W}^{S}\left(f, g_{1}\right)$ is constant over $(a, b)$. Clearly, $\xi_{1}(a)=\xi_{2}(a)$ and $\xi_{1}(b) \geq \xi_{2}(b)$, then

$$
\xi_{1}^{\prime}=\frac{\xi_{1}(b)-\xi_{1}(a)}{b-a} \geq \frac{\xi_{2}(b)-\xi_{2}(a)}{b-a}=\xi_{2}^{\prime} .
$$

The last equality needs justification. For this purpose, let us assume the contrary, i.e. $D^{+}\left(\xi_{2}\right)$ or $D^{-}\left(\xi_{2}\right)$ is not empty. But such an event contradicts monotonicity of $x \mapsto S\left(f^{\prime}\left(x_{0}\right), g_{2}(x), x\right)$.

Now, we recall a natural class of test function. Let us set $Q=(0, T) \times \Omega$, where $\Omega$ is an open interval and $T>0$. Let $A_{P}(Q)$ be the set of all admissible functions $\psi$ on $Q$ in the sense of [14] i.e., $\psi$ is of the form

$$
\psi(x, t)=f(x)+g(t), f \in C_{P}^{2}(\Omega) \equiv C_{P}(\Omega) \cap C^{2}(\Omega), g \in C^{1}(0, T) .
$$


Definition 4.4. A continuous real-valued function u on $Q$ is a (viscosity) subsolution of

$$
v_{t}=a\left(v_{x}, v, x\right)\left(W_{p}^{\prime}\left(v_{x}, x\right)_{x}+S\left(v_{x}, v, x, t\right)\right),
$$

with periodic boundary conditions in $Q$, when $S$ is Lipschitz continuous, if

$$
\psi_{t}(\hat{t}, \hat{x})-a\left(\psi_{x}(\hat{t}, \hat{x}), u(\hat{t}, \hat{x}), \hat{t}, \hat{x}\right)\left(\Lambda_{W}^{S(\hat{t})}(\psi(\hat{t}), u(\hat{t}), \hat{x})\right) \leq 0,
$$

whenever $(\psi,(\hat{t}, \hat{x})) \in A_{P}(Q) \times Q$ fulfills

$$
\max _{Q}(u-\psi)=(u-\psi)(\hat{t}, \hat{x})
$$

Here, $\psi(\hat{t})$ is a function on $\Omega$ defined by $\psi(\hat{t})=\psi(\hat{t}, \cdot)$. Function $\psi$, satisfying (4.12) is called a test function of $u$ at $(\hat{t}, \hat{x})$.

A (viscosity) supersolution is defined by replacing max with min in (4.12) and the inequality (4.11) with the opposite one. If $u$ is both a sub-and supersolution, it is called a viscosity solution or a generalized solution. Hereafter, we avoid using the word viscosity.

By Comparison Theorem, see [16, Theorem 2.12], it is easy to see that $\psi \in A_{P}(Q)$ is a subsolution in $Q$ if (and only if) $\psi$ satisfies

$$
\left.\psi_{t}(t, x)-a\left(\psi_{x}(t, x), \psi(t, x), t, x\right)\left(\Lambda_{W}^{S(\psi(\cdot), \psi(\cdot), \cdot, t)}\right)(\psi, \psi, x)\right) \leq 0
$$

for all $(t, x) \in Q$.

\subsection{Variational solutions are viscosity solutions, when the vertex is pre- served}

We will assume in this section that $\Gamma(t)$ is a family of gently bent rectangles, which is a variational solution to (1.1) and $u$ is the corresponding profile function. We want to show that: (a) $u$ is a subsolution to (3.11); (b) $u$ is a supersolution to (3.11). We shall see that this is not always possible, because a restriction appears. This is explained below.

Theorem 4.2. Let us suppose that $(\Gamma(t), \xi(t))$ is a family of gently bent rectangles which is a variational solution to (1.1) and $u$ is the corresponding profile function. Then, $u$ is a viscosity solution in the sense of Definition 4.4 if and only if (4.16) holds.

Condition (4.16) is equivalent to the preservation of verteces of $\Gamma(t)$. Its disadvantage is that it depends of $\Gamma$ itself. At the end of this section, we will present in Lemma 4.6 a simple sufficient condition for (4.16) to hold independently of $\Gamma$.

We start with the above task (a), i.e. we will show that profile function $u$ is a viscosity subsolution to eq. (3.11). Thus, without the loss of generality, we may restrict our attention to $U_{\mathrm{I}}^{0} \cup U_{\mathrm{I}}^{0} \cup U_{\mathrm{II}}^{0}$, because $U_{\mathrm{IV}}^{0}$ (resp. $U_{\mathrm{V}}^{0}$ ) may be treated like $U_{\mathrm{II}}^{0}$ (resp. $U_{\mathrm{I}}^{0}$ ). For a given test function $\psi$, we have to investigate various possibilities of $(\hat{x}, \hat{t}) \in \operatorname{argmax}(u-\psi)$ :

(1) $(\hat{x}, \hat{t}) \in\left[-k_{\mathrm{I}}(\hat{t}), k_{\mathrm{I}}(\hat{t})\right]$

(2) $(\hat{x}, \hat{t}) \in\left(k_{\mathrm{I}}(\hat{t}), k_{\mathrm{II}}^{l}(\hat{t})\right)$; 
(3) $(\hat{x}, \hat{t}) \in\left[k_{\mathrm{II}}^{l}(\hat{t}), k_{\mathrm{II}}^{r}(\hat{t})\right] \backslash\left\{k_{\mathrm{II}}^{v}(\hat{t})\right\}$;

(4) $\hat{x}=k_{\mathrm{II}}^{v}(\hat{t})$.

The remaining cases are dealt with as those above.

We will investigate situations described in (1)-(4), one by one.

Lemma 4.2. Let us suppose that $\psi$ is a test function satisfying $\max _{Q}(u-\psi)=(u-\psi)(\hat{t}, \hat{x})$ and $(\hat{x}, \hat{t}) \in\left[-k_{I}(\hat{t}), k_{I}(\hat{t})\right]$, then inequality (4.11) holds.

Proof. This case was in fact considered in the proof of Theorem 12 in [16, §5]. It will not be repeated here since we will present a similar technique below anyway.

Lemma 4.3. Let us suppose that $\psi$ is a test function satisfying $\max _{Q}(u-\psi)=(u-\psi)(\hat{t}, \hat{x})$ and $(\hat{x}, \hat{t}) \in\left(k_{I}(\hat{t}), k_{I I}^{l}(\hat{t})\right)$, then inequality (4.11) holds.

Proof. On interval $\left(k_{\mathrm{I}}(\hat{t}), k_{\mathrm{II}}^{l}(\hat{t})\right)$, the profile function $u$ avoids the singular slopes due to the special construction of $W$. As a result, this case is classical and left to the interested reader.

Lemma 4.4. Let us suppose that $\psi$ is a test function, satisfying $\max _{Q}(u-\psi)=(u-\psi)(\hat{t}, \hat{x})$ and $\hat{x} \in\left[k_{\text {III }}^{l}(\hat{t}), k_{\text {III }}^{r}(\hat{t})\right] \backslash\left\{k_{\text {III }}^{v}(\hat{t})\right\}$, then inequality (4.11) holds.

Proof, Step 1. We have two possibilities for $\hat{x}$, which we will consider separately:

(a) $\hat{x} \in\left(k_{\mathrm{II}}^{l}(\hat{t}), k_{\mathrm{II}}^{r}(\hat{t})\right) \backslash\left\{k_{\mathrm{II}}^{v}(\hat{t})\right\}$,

(b) $\hat{x}=k_{\mathrm{II}}^{l}(\hat{t})$ or $\hat{x}=k_{\mathrm{II}}^{r}(\hat{t})$.

Case (b) has its subcases:

$(\alpha) \hat{x}$ is such that $l_{1}(\hat{t})=\Psi(\hat{x}, u(\hat{x}, \hat{t}))$ is a tangency point,

$(\beta) \hat{x}$ is such that $l_{1}(\hat{t})=\Psi(\hat{x}, u(\hat{x}, \hat{t}))$ is a matching point.

In each of those cases we have a number of possibilities how $\psi(x, t)=f(x)+g(t)$ touches $u$ at $(\hat{x}, \hat{t})$. Regardless of the position of $\hat{x}$, we have the following options for $\Lambda_{W}^{Z}(f)$ :

(i) $\Lambda_{++}(R(f, \hat{x}))$, (ii) $\Lambda_{-+}(R(f, \hat{x}))$, (iii) $\Lambda_{+-}(R(f, \hat{x}))$, (iv) $\Lambda_{--}(R(f, \hat{x}))$.

In addition, the behavior of $g^{\prime}(\hat{t})$ will depend on the position of $\hat{x}$, i.e. case (a) differs from (b).

The geometry of the problem is such that for $\hat{x} \in\left[k_{\mathrm{II}}^{l}, k_{\mathrm{II}}^{v}(t)\right)$, for profile function $u$, we have

$$
\Lambda_{W}^{S}(u, u, x)=\Lambda_{--}\left(\left[k_{\mathrm{II}}^{l}(\hat{t}), k_{\mathrm{II}}^{v}(\hat{t})\right]\right) .
$$

However, we have to compare $\Lambda_{--}\left(\left[k_{\mathrm{II}}^{l}(\hat{t}), k_{\mathrm{II}}^{v}(\hat{t})\right]\right.$ with the definition of the variational solution. We see that if $\hat{x} \in\left(k_{\mathrm{II}}^{l}(\hat{t}), k_{\mathrm{II}}^{v}(\hat{t})\right)$, then

$$
Z_{1}(x)=\int_{k_{\mathbb{I I}}^{l}(\hat{t})}^{x} \sigma(\Psi(s, s)) d s=\int_{k_{\mathbb{I I}}^{l}(\hat{t})}^{x} \sigma\left(\frac{s}{\sqrt{2}}, L_{1}(\hat{t})\right) d s .
$$

On the other hand, for $\hat{x} \in\left(k_{\mathrm{II}}^{v}(\hat{t}), k_{\mathrm{II}}^{r}(\hat{t})\right)$, we have

$$
Z_{2}(x)=\int_{k_{\text {II }}^{v}(\hat{t})}^{x} \sigma(\Psi(s,-s)) d s=\int_{k_{\text {II }}^{v}(\hat{t})}^{x} \sigma\left(R_{1}, L_{1}-\frac{s}{\sqrt{2}}\right) d s .
$$

We notice that the minimization problem underlying the definition of the variational solution, see (2.4) and (2.7), $\min \left\{\int_{r_{1}}^{R_{1}}\left|\sigma\left(t, s, L_{1}\right)-\xi_{x}\right|^{2} d s: \xi \in H^{1}\left(\left(r_{1}, R_{1}\right)\right), \xi \in\left[-\gamma_{\Lambda}, \gamma_{\Lambda}\right], \xi\left(r_{1}\right)=-\gamma_{\Lambda}, \xi\left(R_{1}\right)=\gamma_{\Lambda}\right\}$, 
for $\hat{x} \in\left(k_{\mathbb{I I}}^{l}, k_{\mathrm{II}}^{v}(t)\right)$, (respectively, $\min \left\{\int_{l_{1}}^{L_{1}}\left|\sigma\left(t, R_{1}, s\right)-\xi_{x}\right|^{2} d s: \xi \in H^{1}\left(\left(l_{1}, L_{1}\right)\right), \xi \in\left[-\gamma_{R}, \gamma_{R}\right], \xi\left(l_{1}\right)=-\gamma_{R}, \xi\left(L_{1}\right)=\gamma_{R}\right\}$,

for $\left.\hat{x} \in\left(k_{\mathrm{II}}^{v}(t), e_{V}^{R}\right)\right)$, coincides with the minimization leading to $\Lambda_{--}\left(\left[k_{\mathrm{II}}^{l}(\hat{t}), k_{\mathrm{II}}^{v}(\hat{t})\right]\right)$ (respectively, $\left.\Lambda_{--}\left(\left[k_{\mathbb{I I}}^{v}(\hat{t}), k_{\mathrm{II}}^{r}(\hat{t})\right]\right)\right)$. This statement is clear for $\hat{x} \in\left(k_{\mathrm{II}}^{l}(\hat{t}), k_{\mathrm{II}}^{v}(\hat{t})\right)$, while an additional change of variables in the integral above is necessary for $\hat{x} \in\left(k_{\mathbb{I I}}^{v}(\hat{t}), k_{\mathbb{I I}}^{r}(\hat{t})\right)$.

Step 2 . We start with case (a). We immediately notice that under these circumstances $u_{t}(\hat{x}, \hat{t})$ exists, hence $g^{\prime}(\hat{t})=u_{t}(\hat{x}, \hat{t})$. Furthermore, we only know that $\hat{x} \in\left(k_{\mathbb{I I}}^{l}(\hat{t}), k_{\mathbb{I I}}^{v}(\hat{t})\right) \cap R(f, \hat{x})$. By Theorem 4.1, we deduce that

$$
\begin{gathered}
\Lambda_{--}\left(\left[k_{\mathbb{I I}}^{l}(\hat{t}), k_{\mathbb{I I}}^{v}(\hat{t})\right]\right) \leq \Lambda_{++}(R(f, \hat{x})), \quad \Lambda_{-+}\left(\left[k_{\mathbb{I I}}^{l}(\hat{t}), k_{\mathbb{I I}}^{v}(\hat{t})\right]\right) \leq \Lambda_{++}(R(f, \hat{x})), \\
\Lambda_{+-}\left(\left[k_{\mathbb{I I}}^{l}(\hat{t}), k_{\mathbb{I I}}^{v}(\hat{t})\right]\right) \leq \Lambda_{++}(R(f, \hat{x})) .
\end{gathered}
$$

Hence, these inequalities imply,

$$
\psi_{t}(\hat{x}, \hat{t})-\Lambda_{W}^{Z}(\psi(\hat{x}, \hat{t}))=u_{t}(\hat{x}, \hat{t})-\Lambda_{W}^{Z}(u(\hat{x}, \hat{t}))+\Lambda_{W}^{Z}(u(\hat{x}, \hat{t}))-\Lambda_{++}(R(f, \hat{x})) \leq 0,
$$

where $Z$ equals $Z_{1}$ or $Z_{2}$ defined above, depending upon the position of $\hat{x}$. The case $\Lambda_{W}^{Z}(f)=$ $\Lambda_{--}(R(f, \hat{x}))$ is possible if and only if $\left[k_{\mathrm{II}}^{l}(\hat{t}), k_{\mathrm{II}}^{v}(\hat{t})\right] \subset R(f, \hat{x})$. If this happens, then Theorem 4.1 (ii) implies that

$$
\psi_{t}(\hat{x}, \hat{t})-\Lambda_{W}^{Z}(\psi(\hat{x}, \hat{t})) \leq 0
$$

holds.

Step 3. We first consider case $(\alpha)$. This means the interfacial point $r_{1}(t)$ moves to the left, $\dot{r}_{1}(\hat{t})<0$ and $\beta\left(\mathbf{n}_{R}\right) \frac{d}{d t} L_{1}(t)=\sigma\left(r_{1}(t), L_{1}(t), t\right)$. This implies that $d_{t}^{+}\left(r_{1}(\hat{t}), \hat{t}\right)=\frac{d}{d t} L_{1}(t)$ and $d_{t}^{-}\left(r_{1}(\hat{t}), \hat{t}\right)=d_{x}^{-}\left(r_{1}(\hat{t}), \hat{t}\right)=\sigma\left(r_{1}(t), L_{1}(t), t\right) / \beta\left(\mathbf{n}_{R}\right)$. As a result $g^{\prime}(\hat{t})=\sigma\left(r_{1}(t), L_{1}(t), t\right) / \beta\left(\mathbf{n}_{R}\right)$ and a reasoning as in Step 2 applies leading to the conclusion that (4.11) holds.

We have to consider case $(\beta)$. According to formula (2.25) in [18],

$$
\operatorname{sgn} \dot{r}_{1}(\hat{t})=\operatorname{sgn}\left(\dot{L}_{1}-\sigma\left(\hat{t}, r_{1}(\hat{t}), L_{1}(\hat{t})\right) .\right.
$$

In any case, $\Lambda_{W}^{Z}(u)=\Lambda_{--}\left(\left[k_{\mathbb{I I}}^{l}(\hat{t}), k_{\mathrm{II}}^{v}(\hat{t})\right]\right)$.

We notice that if $\dot{r}_{1}(\hat{t})<0$, then

$$
u_{t}^{+}(\hat{x}, \hat{t})=\sigma\left(t, r_{1}(\hat{t}), L_{1}(\hat{t}) / \beta\left(\mathbf{n}_{R}\right)<\dot{L}_{1}(\hat{t}) / \beta\left(\mathbf{n}_{R}\right)=u_{t}^{-}(\hat{x}, \hat{t}) .\right.
$$

Thus

$$
g^{\prime}(\hat{t}) \in\left[\sigma \left(t, r_{1}(\hat{t}), L_{1}(\hat{t}) / \beta\left(\mathbf{n}_{R}\right), \dot{L}_{1}(\hat{t}) / \beta\left(\mathbf{n}_{R}\right) .\right.\right.
$$

Hence,

$$
\begin{aligned}
\psi_{t}(\hat{x}, \hat{t})-\Lambda_{W}^{Z}(\psi)(\hat{x}, \hat{t}) & \leq L_{1}(\hat{t}) / \beta\left(\mathbf{n}_{R}\right)-\Lambda_{W}^{Z}(u)(\hat{x}, \hat{t})+\Lambda_{W}^{Z}(u)(\hat{x}, \hat{t})-\Lambda_{W}^{Z}(\psi)(\hat{x}, \hat{t}) \\
& =0+\Lambda_{--}\left(\left[k_{\mathrm{II}}^{l}(\hat{t}), k_{\mathrm{II}}^{v}(\hat{t})\right]\right)-\Lambda_{W}^{Z}(\psi)(\hat{x}, \hat{t}) \leq 0 .
\end{aligned}
$$

The last inequality is a consequence of Theorem 4.1, because $\Lambda_{W}^{Z}(\psi)$ may be one of the following quantities,

$$
\Lambda_{++}(R(f, \hat{x})), \quad \Lambda_{-+}(R(f, \hat{x})), \quad \Lambda_{+-}(R(f, \hat{x})), \quad \Lambda_{--}(R(f, \hat{x})) .
$$


If we deal with the last quantity, then $R(f, \hat{x}) \subset\left[k_{\mathbb{I I}}^{l}(\hat{t}), k_{\mathrm{II}}^{v}(\hat{t})\right]$.

We observe that if $\dot{r}_{1}(\hat{t})>0$, then there are no test functions.

We come to the study of the vertex of $\Gamma$ in the first quadrant of the plane. It is done separately from other cases. Here, we need additional information on the kinetic coefficient $\beta$.

Lemma 4.5. Let us suppose that $\psi$ is a test function satisfying $\max _{Q}(u-\psi)=(u-\psi)(\hat{t}, \hat{x})$ and $\hat{x}=k_{\text {III }}^{v}(\hat{t})$. Then, inequality (4.11) holds if and only if (4.16) is satisfied.

Proof. We are going to show that a variational solution is a viscosity subsolution. Let us take a test function $\psi(x, t)=f(x)+g(t)$ such that

$$
\max (u-\psi)=(u-\psi)\left(k_{\mathrm{II}}^{v}(t), t\right)=0 .
$$

For the sake of consistency, we shall write $(\hat{x}, \hat{t})$ in place of $\left(k_{\mathbb{I I}}^{v}(t), t\right)$.

The profile function $u$ takes the following form in a neighborhood of $(\hat{x}, \hat{t})$

$$
u(x, t)=y(t)-|x-x(t)|,
$$

where $\hat{x}=x(\hat{t})$ and $u(\hat{x}, \hat{t})=y(\hat{t})$. The vertex of $\Gamma(t)$ is determined as the intersection point of two lines,

$$
\begin{aligned}
& y=x+A(t), \\
& y=-x+B(t) .
\end{aligned}
$$

Thus, we can see that the intersection point has the coordinates

$$
(x, y)=\frac{1}{2}(B-A, B+A) \text {. }
$$

Here, $A^{\prime}(t)$ is the vertical velocity of the facet with the slope $p=1$, and $B^{\prime}(t)$ is the vertical velocity of the facet with the slope $p=-1$. That is, we can relate them to the normal velocities of the facets,

$$
A^{\prime}(t)=\frac{\sqrt{2}}{2 \beta_{R}}\left(f_{r_{1}}^{R_{1}} \sigma\left(t, s, L_{1}\right) d s-\frac{2 \gamma\left(\mathbf{n}_{\Lambda}\right)}{R_{1}-r_{1}}\right), \quad B^{\prime}(t)=\frac{\sqrt{2}}{2 \beta_{\Lambda}}\left(f_{l_{1}}^{L_{1}} \sigma\left(t, R_{1}, s\right) d s-\frac{2 \gamma\left(\mathbf{n}_{R}\right)}{L_{1}-l_{1}}\right) .
$$

We also have to determine the restrictions on the test function resulting from (4.14) and (4.15). We notice that for $x \geq \hat{x}$ we have,

$$
u(x, t)-\psi(x, t)=y(t)+x(t)-g(t)-f(x)-x \leq 0
$$

and for $x \leq \hat{x}$ we have,

$$
u(x, t)-\psi(x, t)=y(t)-x(t)-g(t)-f(x)+x \leq 0 .
$$

Thus, we infer that $f^{\prime}(\hat{x}) \in[-1,1]$ and $g^{\prime}(\hat{t})$ is in the interval with endpoints

$$
\dot{y}(\hat{t})+\dot{x}(\hat{t})=B^{\prime}(\hat{t}), \quad \dot{y}(\hat{t})-\dot{x}(\hat{t})=A^{\prime}(\hat{t}) .
$$


Keeping this in mind we consider $f$, which is not faceted at $\hat{x}$, i.e. $\left|f^{\prime}(\hat{x})\right|<1$. We have to check that

$$
\psi_{t}-a\left(x, t, \psi, \psi_{x}\right) \sigma\left(t, R_{1}(t), L_{1}(t)\right) \leq 0
$$

holds. Since $g^{\prime}(\hat{t})=\theta A^{\prime}(\hat{t})+(1-\theta) B^{\prime}(\hat{t})$ for $\theta \in[0,1]$, this inequality is equivalent to

$$
\begin{aligned}
& \theta \frac{\sqrt{2}}{2 \beta_{R}}\left(f_{r_{1}}^{R_{1}} \sigma\left(t, s, L_{1}\right) d s-\frac{2 \gamma\left(\mathbf{n}_{\Lambda}\right)}{R_{1}-r_{1}}\right)+(1-\theta) \frac{\sqrt{2}}{2 \beta_{\Lambda}}\left(f_{l_{1}}^{L_{1}} \sigma\left(t, R_{1}, s\right) d s-\frac{2 \gamma\left(\mathbf{n}_{R}\right)}{L_{1}-l_{1}}\right) \\
\leq & \frac{\sigma\left(t, R_{1}(t), L_{1}(t)\right)}{\beta(\mathbf{n})},
\end{aligned}
$$

where $\theta \in[0,1]$ and $\mathbf{n}$ is between $\mathbf{n}_{R}$ and $\mathbf{n}_{\Lambda}$.

If $f$ is faceted, then this case reduces to the situation considered Lemma 4.4.

We may sum up the the above results as follows.

Corollary 4.1. If $\Gamma(t)$ is a family of gently bent rectangles which is a variational solution to (1.1) and $u$ is the corresponding profile function. Then, $u$ is a viscosity subsolution if and only if (4.16) holds.

Proof. Combining Lemmata from 4.2 to 4.5 shows that $u$ is a subsolution.

After these preparations we will embark on the proof of Theorem 4.2. It remains to check that $u$ is a supersolution. It is easier, than showing that $u$ is a subsolution, because for any test function $\psi$ due to the form of the profile function $u$, see (4.15), the minimum of $u-\psi$ may not occur at $k_{\mathbb{I I}}^{v}(\hat{t}, \hat{t})$. Thus we have the following possibilities for $(\hat{x}, \hat{t}) \in \operatorname{argmin}(u-\psi)$ :

(1) $(\hat{x}, \hat{t}) \in\left[-k_{\mathrm{I}}(\hat{t}), k_{\mathrm{I}}(\hat{t})\right]$;

(2) $(\hat{x}, \hat{t}) \in\left(k_{\mathrm{I}}(\hat{t}), k_{\mathrm{II}}^{l}(\hat{t})\right)$;

(3) $(\hat{x}, \hat{t}) \in\left[k_{\mathrm{II}}^{l}(\hat{t}), k_{\mathrm{II}}^{r}(\hat{t})\right] \backslash\left\{k_{\mathrm{II}}^{v}(\hat{t})\right\}$.

Case (1) is the content of [16, Theorem 12]. Case (2) is easy and left to the reader. We will consider (3). We first notice that due to the geometry of the graph of $u$ there is no test function $\psi$ such that $\left.\min (u-\psi)=(u-\psi)\left(k_{\mathrm{II}}^{l}(\hat{t}), \hat{t}\right)\right)$. Thus, we consider $(\hat{x}, \hat{t}) \in$ $\left(k_{\mathrm{II}}^{l}(\hat{t}), k_{\mathrm{II}}^{r}(\hat{t})\right) \backslash\left\{k_{\mathrm{II}}^{v}(\hat{t})\right\}$. Immediate conclusions are that $\Lambda_{W}^{Z}(u)=\Lambda_{--}\left(\left[k_{\mathrm{II}}^{l}(\hat{t}), k_{\mathrm{II}}^{v}(\hat{t}]\right)\right)$, (or $\Lambda_{W}^{Z}(u)=\Lambda_{--}\left(\left[k_{\mathbb{I I}}^{v}(\hat{t}), k_{\mathrm{II}}^{r}(\hat{t})\right]\right), g^{\prime}(\hat{t})=\dot{L}_{1}(\hat{t})$ and $\Lambda_{W}^{Z}(f)=\Lambda_{--}(R(f, \hat{x}))$. Moreover, by Theorem 4.1 (i) $\Lambda_{--}\left(\left[k_{\mathrm{II}}^{l}(\hat{t}), k_{\mathrm{II}}^{v}(\hat{t}]\right) \leq \Lambda_{--}(R(f, \hat{x}))\right.$. As a result (4.11) holds. The proof is complete.

We have seen that inequality (4.16) is necessary and sufficient for variational solutions to be viscosity solutions, but we would like to discover a simpler sufficient condition. Here one such a result.

Lemma 4.6. Let us suppose that

$$
\frac{1}{\beta(\mathbf{n})} \geq \frac{\sqrt{2}}{2}\left(\frac{\theta}{\beta\left(\mathbf{n}_{R}\right)}+\frac{1-\theta}{\beta\left(\mathbf{n}_{\Lambda}\right)}\right) \quad \text { for all } \theta \in[0,1]
$$

or

$$
\max \left\{R_{1}-r_{1}, L_{1}-l_{1}\right\} \text { is sufficiently small, }
$$

then condition (4.16) is satisfied. 
Proof. By monotonicity of $\sigma$ we obtain

$$
\begin{aligned}
& \frac{\sqrt{2}}{4 \beta_{R}}\left(f_{r_{1}}^{R_{1}} \sigma\left(t, s, L_{1}\right) d s-\frac{2 \gamma\left(\mathbf{n}_{\Lambda}\right)}{R_{1}-r_{1}}\right)+\frac{\sqrt{2}}{4 \beta_{\Lambda}}\left(f_{l_{1}}^{L_{1}} \sigma\left(t, R_{1}, s\right) d s-\frac{2 \gamma\left(\mathbf{n}_{R}\right)}{L_{1}-l_{1}}\right) \\
\leq & \sigma\left(t, R_{1}(t), L_{1}(t)\right) \frac{\sqrt{2}}{4}\left(\frac{1}{\beta\left(\mathbf{n}_{R}\right)}+\frac{1}{\beta\left(\mathbf{n}_{\Lambda}\right)}\right)-\frac{\sqrt{2}}{2}\left(\frac{\gamma\left(\mathbf{n}_{\Lambda}\right)}{R_{1}-r_{1}}+\frac{\gamma\left(\mathbf{n}_{R}\right)}{L_{1}-l_{1}}\right) \\
\leq & \frac{\sigma\left(t, R_{1}(t), L_{1}(t)\right)}{\beta(\mathbf{n})} .
\end{aligned}
$$

We say that $R_{1}-r_{1}$ and $L_{1}-l_{1}$ are sufficiently small if $A^{\prime} \leq 0$ and $B^{\prime} \leq 0$.

\section{A comparison principle}

Since equation (3.11) does not fit the framework of [16], we may not apply directly the comparison principle to problem (3.11). Such a comparison principle would be of an independent interest. However, here a simpler result would do the job of proving the uniqueness of variational solutions to equations (1.1).

\subsection{An adjustment of the known result}

In this section, we shall establish a comparison principle for our singular diffusion equation

$$
u_{t}=a\left(x, u, u_{x}\right)\left(W_{p}\left(u_{x}, x\right)_{x}+\tilde{\sigma}(u, x, t)\right),
$$

which we derived in previous sections. A new aspect of the problem is that $a$ and $\tilde{\sigma}$ depend on $u$ explicitly. This makes the problem more involved. Instead of discussing a general situation we rather establish a comparison principle for a special function called a profile function of a gently bent rectangle.

We recall several important properties of (5.1) which are obviously fulfilled for our singular diffusion equations by replacing the arclength parameter $s$ by $x$ and $v$ by $u$; $\tilde{\sigma}$ is replaced by $\sigma$. Let $2 \pi L$ be the length of the reference manifold. We consider (5.1) with a periodic boundary condition with period $2 \pi L$. The domain of definition is divided into $U_{\mathrm{I}}, U_{\mathrm{II}}, U_{\mathrm{II}}, U_{\mathrm{IV}}, U_{\mathrm{V}}$, see (3.13)-(3.16). While $\tilde{\sigma}$ may always depend on $u$ and $x$, we note that in $U_{\mathrm{I}}, U_{\mathbb{I I}}, U_{\mathrm{V}} a$ is a positive function depending only on $u_{x}$ and that $W$ is of the form

$$
\begin{array}{ll}
W(p, x)=\gamma_{\Lambda}|p| & \text { in } U_{\mathrm{I}} \\
W(p, x)=\gamma_{\Lambda}|p-1|+\gamma_{R}|p+1| & \text { in } U_{\mathrm{III}} \\
W(p, x)=\gamma_{R}|p| & \text { in } U_{\mathrm{V}} .
\end{array}
$$

In particular, in these regions, the equation is the same as we have studied in [16] except that $\tilde{\sigma}$ depends on $u$ explicitly. Our $a$ is always smooth (and globally Lipschitz in $u_{x}$ and $x$ ) and by construction $\partial a / \partial u$ is bounded independently of $x, u, u_{x}$. For $\tilde{\sigma}$ we assume that it is $C^{1}$ and $\partial \tilde{\sigma} / \partial u$ is bounded from below independent of $u, x, t$ to avoid unnecessary complexity. We further impose a vertex preserving condition for the mobility which reflects the condition for $a$. (This is the condition that the function $-\left|x-x_{0}\right|$ in $U_{\text {III }}$ keeps its vertex when it evolves by $u_{t}=a\left(u_{x}\right)$.) 
We rather consider a special class of functions when we consider a solution of (5.1). We start with one connected component of $U_{\mathrm{I}}$, say $U_{\mathrm{I}}^{0}$ and list connected components of $U_{\mathbb{I}}, \ldots, U_{\mathrm{V}}$ next to each other clockwise and name them $U_{\mathbb{I}}^{0}, \ldots, U_{\mathrm{V}}^{0}$. We name connected components further clockwise like $U_{\mathrm{I}}^{1}, U_{\mathrm{II}}^{1}, \ldots, U_{\mathrm{I}}^{1}, U_{\mathrm{II}}^{2}, \ldots, U_{\mathrm{V}}^{2}, U_{\mathrm{N}}^{3}, \ldots, U_{\mathrm{II}}^{0}, U_{\mathrm{I}}^{0}$.

Theorem 5.1 (Comparison principle). Let $u$ and $v$ be profile functions of a gently bent rectangle at each $t \in[0, T]$ and $u, v \in C(\mathbb{T} \times[0, T])$. Assume that $u$ and $v$ are respectively a sub-and supersolution of (5.1) in $\mathbb{T} \times(0, T)$. Assume that one of $u$ and $v$ is $C^{1}$ in time on facets and facet ends move continuously in time. Then $u \leq v$ in $\mathbb{T} \times[0, T]$, provided that $u \leq v$ at $t=0$.

Proof. We take a conventional strategy to attack the problem when the Hamiltonian itself depends on the unknown. Let $M$ be a number greater than 1 such that, see (4.2),

$$
\frac{\partial}{\partial u}(a \sigma) \leq(M-1) \quad \text { for all } \quad(x, t, u, p) \in \mathbb{T} \times(0, T] \times \mathbb{R} \times \mathbb{R} .
$$

We may find such $M$ because $u$ and $v$ are bounded on $\mathbb{T} \times(0, T)$ and by assumption $\sigma \in C^{1}$.

We consider $U=e^{-2 M t} u, V=e^{-2 M t} v$. Assume that the conclusion were false, in other words

$$
m=\max _{\mathbb{T} \times[0, T]}\{U(x, t)-V(x, t)\}>0 .
$$

Since $u \leq v$ at $t=0$, the maximum is only attained for $t>0$. We set

$$
\hat{t}=\sup \left\{t_{\max } \mid \max _{\mathbb{T}}(U-V)\left(\cdot, t_{\max }\right)=m\right\} \in(0, T] .
$$

We may assume that there is no $t_{\max }$ other than $\hat{t}$ by multiplying $e^{M t}$ with $U$ and $V$. We still denote $e^{M t} U$ and $e^{M t} V$ by $U$ and $V$ respectively, i.e. $U=e^{-M t} u, V=e^{-M t} v$. Then $U$ solves

$$
U_{t}+M U=e^{-M t} a\left(x, e^{M t} U, e^{M t} U_{x}\right)\left\{W_{p}\left(e^{M t} U_{x}, x\right)_{x}+\sigma\left(e^{M t} U, x, t\right)\right\}
$$

since $u$ solves (5.1) in a formal level. We may assume not only that

$$
m=\max _{\mathbb{T} \times[0, T]}(U-V)>0
$$

but also

$$
\begin{gathered}
(U-V)(x, t)<m \text { for } x \in \mathbb{T}, t<\hat{t} \\
m=\max _{\mathbb{T}}(U-V)(\cdot, \hat{t}) .
\end{gathered}
$$

We shall divide the situation into two cases.

Case I. $m$ is attained at interior points of faceted regions of both functions $U(\cdot, \hat{t})$ and $V(\cdot, \hat{t})$.

Case II. $m$ is attained only outside of the interior of faceted regions of both functions $U(\cdot, \hat{t})$ and $V(\cdot, \hat{t})$. 
Another seemingly existing case can not happen because of the geometry of profile functions.

We begin with Case I. Since we have assumed that motion of the facets in time in $C^{1}$ and the facet ends move continuously in time for one of $U$ and $V$, the argument is very simple. To fix attention we assume that $U$ is this function. (The case that $V$ is such a function can be treated in the same way.) Assume that the maximum is attained in an interior point $\hat{x}$ of facets. Then it is either in $U_{\mathrm{I}}, U_{\mathrm{II}}, U_{\mathrm{V}}$ because of the geometry of profile functions. All cases can be treated similarly so we only consider the case that such facets are in $U_{\mathbb{I I}}^{0}$. Then the function $a$ is independent of $u$ and $x$. We rearrange (5.3) in $U_{\text {III }}$ to get

$$
U_{t}=e^{-M t} a\left(e^{M t} U_{x}\right)\left\{W_{p}\left(e^{M t} U_{x}\right)\right\}_{x}+e^{-M t} a\left(e^{M t} U_{x}\right) \tilde{\sigma}\left(e^{M t} U, x, t\right)-M U .
$$

By the choice of $M$ the term $e^{-M t} a \sigma-M U+U$ is nonincreasing in $U$.

We want to regard $U$ as a test function of a supersolution $V$. We notice that $\min (V-U)=$ $-\max (U-V)=-m<0$. But $U$ lacks the structure and regularity of functions in $A_{P}(\Omega)$. Strictly speaking, we have to find $\psi \in A_{P}$. We set,

$$
\tilde{f}(x):=U(x, \hat{t}), \quad g(t):=U(\hat{x}, t),
$$

Of course, $\tilde{f}+g=U$ on $R(U, \hat{x}) \times\{\hat{t}\}$. Since $U_{t}$ exists on $R(U, \hat{x})$, we notice that $\min (V-$ $(\tilde{f}+g))$ is attained at $t=\hat{t}$. But now, $\tilde{f}$ lacks the necessary smoothness. However, it is easy to see that regardless of the configuration of the endpoints of $R(U, \hat{x})$ and $R(V, \hat{x})$ then we can mollify $\tilde{f}$ to get $f$ so that, $R(f, \hat{x})=R(\tilde{f}, \hat{x})$ and

$$
\Lambda_{W}^{Z}(f)=\Lambda_{W}^{Z}(\tilde{f})=\Lambda_{W}^{Z}(U) .
$$

Thus, keeping this in mind we have the following inequality at $\hat{x}$

$$
V+U_{t} \geq e^{-M \hat{t}} a\left(u_{x}\right) \Lambda_{W}^{S}(U, V, x, \hat{t})
$$

at $\hat{x}$,

$$
S\left(f^{\prime}, g, x, t\right)=\tilde{\sigma}\left(e^{t M} g, x, t\right)-(M-1) \frac{g}{a\left(f^{\prime}(x)\right)} .
$$

Due to a (5.2) function $S$ is decreasing with respect to $g$.

Here, we invoke the assumption that the facet does not break on the profile function $U$, i.e. $\Lambda_{W}^{S}(U, U, x, \hat{t})$ is constant over $R(U, \hat{x})$.

Since $U$ is a subsolution and $V$ is a supersolution, we also have

$$
V+U_{t} \leq e^{-M \hat{t}} a\left(u_{x}\right) \Lambda_{W}^{S}(U, U, x, t)
$$

at $\hat{x}$. These two inequalities imply that

$$
0>V-U \geq e^{-M \hat{t}} a\left(u_{x}(\hat{x}, \hat{t})\right)\left(\Lambda_{W}^{S}(U, V, x, t)-\Lambda_{W}^{S}(U, U, x, t)\right) .
$$

Now, we recall the monotonicity property of $S$, stated above and Lemma 4.1, implying that the RHS is positive, which leads to a contradiction.

It remains to prove Case II. We may assume that a maximum is attained in $U_{\mathrm{I}}^{0}, U_{\mathrm{II}}^{0}$ and $U_{\mathrm{II}}^{0}$. Let $y_{u}(s)$ be the right end of the faceted region of $V(\cdot, s)$ in $U_{\mathrm{I}}^{0}$ and let $x_{u}(t)$ be the left end of 
the faceted region of $U(\cdot, t)$ in $U_{\mathbb{I I}}^{0}$. The function $U(\cdot, t)$ is strictly increasing from the right end of the faceted region of $U(\cdot, t)$ to $x_{u}$ and a similar monotonicity holds for $V(\cdot, s)$. We set

$$
w(x, y, t, s)=U(x, t)-V(y, s) .
$$

We are interested in maximizers of $w$ in $|x-y| \leq \delta,|t-s| \leq \delta$ for sufficiently small $\delta>0$. We may assume that there are no maximizers in the interior of faceted regions since otherwise it can be reduced to Case I by shifting $U(x+h, t+k)$ slightly. By the monotone property of $U(\cdot, t), V(\cdot, s)$ we may easily conclude that,

$$
\arg \max w \subset\{y \leq x\} \cap\left\{x \leq x_{u}(t)\right\} \cap\left\{y \geq y_{v}(s)\right\}
$$

in $(\mathbf{T} \times[0, T])^{2}$ for $|x-y| \leq \delta,|t-s| \leq \delta$. This observation shows that,

$$
\arg \max \Phi \subset\{y \leq x\} \cap\left\{x \leq x_{u}(t)\right\} \cap\left\{y \geq y_{v}(s)\right\}
$$

for $\Phi=w-\alpha|x-y|^{2}-\beta|t-s|^{2}$ provided that $\alpha$ and $\beta$ is taken sufficiently large. Since we have assumed (iv) and (vi) in Definition 4.3 about the profile functions, one can argue in the same way as above that we need to discuss the following equation

$$
U_{t}+M U=e^{-M t} a\left(x, e^{M t} U, e^{M t} U_{x}\right) \sigma\left(e^{M t} U, x, t\right)
$$

to get a contradiction.

Indeed let $\left(x_{\alpha \beta}, y_{\alpha \beta}, s_{\alpha \beta}, r_{\alpha \beta}\right)$ be a maximizer of $\Phi$, i.e.

$U(x, t)-V(y, s)-\alpha|x-y|^{2}-\beta|t-s|^{2} \leq U\left(x_{\alpha \beta}, t_{\alpha \beta}\right)-V\left(y_{\alpha \beta}, s_{\alpha \beta}\right)-\alpha\left|x_{\alpha \beta}-y_{\alpha \beta}\right|^{2}-\beta\left|s_{\alpha \beta}-t_{\alpha \beta}\right|^{2}$.

By setting $y=y_{\alpha \beta}, s=s_{\alpha \beta}$, we immediately see that $\alpha\left|x-y_{\alpha \beta}\right|^{2}+\beta\left|t-s_{\alpha \beta}\right|^{2}$ is the test function of $U$ at $\left(x_{\alpha \beta}, t_{\alpha \beta}\right)$ touching it from above. We thus observe that

$$
2 \beta\left(t_{\alpha \beta}-s_{\alpha \beta}\right)+M U\left(x_{\alpha \beta}, t_{\alpha \beta}\right) \leq H\left(x_{\alpha \beta}, t_{\alpha \beta}, U\left(x_{\alpha \beta}, t_{\alpha \beta}\right), 2 \alpha\left(x_{\alpha \beta}-y_{\alpha \beta}\right)\right)
$$

with $H(x, t, r, p)=e^{-M t} a\left(x, e^{M t} r, e^{M t} p\right) \sigma\left(e^{M t} r, x, t\right)$. Similarly, for $V$ we have

$$
2 \beta\left(t_{\alpha \beta}-s_{\alpha \beta}\right)+M V\left(y_{\alpha \beta}, s_{\alpha \beta}\right) \geq H\left(y_{\alpha \beta}, s_{\alpha \beta}, V\left(y_{\alpha \beta}, s_{\alpha \beta}\right), 2 \alpha\left(x_{\alpha \beta}-y_{\alpha \beta}\right)\right) .
$$

Subtracting the second one from the first in equality yields

$$
\begin{aligned}
M\left(U\left(x_{\alpha \beta}, t_{\alpha \beta}\right)-V\left(y_{\alpha \beta}, s_{\alpha \beta}\right)\right) & \leq H\left(x_{\alpha \beta}, t_{\alpha \beta}, U\left(x_{\alpha \beta}, t_{\alpha \beta}\right), 2 \alpha\left(x_{\alpha \beta}-y_{\alpha \beta}\right)\right) \\
& -H\left(x_{\alpha \beta}, t_{\alpha \beta}, V\left(y_{\alpha \beta}, s_{\alpha \beta}\right), 2 \alpha\left(x_{\alpha \beta}-y_{\alpha \beta}\right)\right) \\
& +H\left(x_{\alpha \beta}, t_{\alpha \beta}, V\left(y_{\alpha \beta}, s_{\alpha \beta}\right), 2 \alpha\left(x_{\alpha \beta}-y_{\alpha \beta}\right)\right) \\
& -H\left(y_{\alpha \beta}, s_{\alpha \beta}, V\left(y_{\alpha \beta}, s_{\alpha \beta}\right), 2 \alpha\left(x_{\alpha \beta}-y_{\alpha \beta}\right)\right) .
\end{aligned}
$$

By the choice of $M$ this implies

$$
\begin{aligned}
U\left(x_{\alpha \beta}, t_{\alpha \beta}\right)-V\left(y_{\alpha \beta}, s_{\alpha \beta}\right) & \leq H\left(x_{\alpha \beta}, t_{\alpha \beta}, V\left(y_{\alpha \beta}, s_{\alpha \beta}\right), 2 \alpha\left(x_{\alpha \beta}-y_{\alpha \beta}\right)\right) \\
& -H\left(y_{\alpha \beta}, s_{\alpha \beta}, V\left(y_{\alpha \beta}, s_{\alpha \beta}\right), 2 \alpha\left(x_{\alpha \beta}-y_{\alpha \beta}\right)\right) .
\end{aligned}
$$

After sending $\alpha \rightarrow \infty, \beta \rightarrow \beta$ and using the continuity of $H$ with respect to $x$ and $t$ we get a contradiction with $m \leq 0$. This is so, because

$$
|H(x, t, r, p)-H(y, s, r, p)| \leq C(|x-y|+|t-s|)(1+|p|)
$$

and since $\alpha\left|x_{\alpha \beta}-y_{\alpha \beta}\right| \rightarrow 0$ which follows by a standard argument. 


\subsection{Uniqueness of solutions to (1.1)}

Here, we address the question of uniqueness of variational solutions to (1.1).

Theorem 5.1. Let us suppose that $\{(\Gamma(t), \xi(t))\}_{t \in[0, T)}$ is a variational solution to (1.1) with $\Gamma(0)=\Gamma_{0}$. If $\Gamma_{0}$ satisfies the geometric condition (3.3), then $\{\Gamma(t)\}_{t \in\left[0, T_{1}\right)}$ is a family of gently bent rectangles. If the kinetic coefficient $\beta(\cdot)$ satisfies (4.16), then $\{\Gamma(t)\}_{t \in[0, T)}$ is a unique solution to (1.1). That is the profile function $u$ of the family $\Gamma(t)$ is the unique viscosity solution to (3.11) with $a$, $W$ and $\tilde{\sigma}$ defined in (3.31), (3.7) and (3.32).

Uniqueness means that if we are given $\left\{\left(\Gamma_{1}(t), \xi_{1}(t)\right)\right\}_{t \in[0, T)}$, another variational solution to (1.1) with $\Gamma_{1}(0)=\Gamma_{0}$, with continuously varying facet endpoints and whose profile function $u_{1}$ is a viscosity solution to (3.11), then $u=u_{1}$ and $\xi=\xi_{1}$. We stress that Lemma 4.6 gives a universal sufficient condition for $u_{1}$, a profile function to be a viscosity solution to (3.11).

Proof. The assumption that $\Gamma_{0}$ satisfies (3.3) implies existence of the reference manifold $\mathcal{M}$ and the possibility of writing $\Gamma(t)$ as a graph of a profile function $u$.

The construction of $\Gamma(t)$, performed in [18], guarantees that the facet endpoints move continuously and their speed is finite. Since we assumed (4.16), then the profile function is not only a viscosity solution to (4.1), but also the verteces are preserved. We notice that we may apply the Comparison Principle 5.1. Thus, $u$ is a unique solution to (4.1). Once we established that $\Gamma(t)=\Gamma_{1}(t)$ for all times, we have to check that $\xi(t)=\xi_{1}$, but this is an easy task, we proceed as in $[22, \S 4]$.

\section{Acknowledgement}

The work of YG was partly supported by Japan Society for the Promotion of Science through the grants Kiban S (26220702), Kiban A (23244015) and Houga (25610025). PG and PR were partially supported by the NCN through 2011/01/B/ST1/01197 grant. The work of YG and PR was performed as a part of the IRSES program ,Flux”.

\section{References}

[1] F.Almgren and L.Wang, Mathematical existence of crystal growth with Gibbs-Thomson curvature effects, J. Geom. Anal., 10 (2000), 1-100.

[2] F.Andreu-Vaillo, V.Caselles, J.Mazon, "Parabolic quasilinear equations minimizing linear growth functionals", Progress in Mathematics, 223. Birkhäuser Verlag, Basel, 2004.

[3] J. W.Barrett, H. Garcke, R. Nürnberg, Finite-element approximation of one-sided Stefan problems with anisotropic, approximately crystalline, Gibbs-Thomson law, Adv. Differential Equations, 18 (2013), no. 3-4, 383-432.

[4] G. Bellettini, V. Caselles, A. Chambolle and M. Novaga, Crystalline mean curvature flow of convex sets, Arch. Ration. Mech. Anal., 179 (2006), 109-152.

[5] G.Bellettini, V.Caselles, A.Chambolle and M.Novaga, The volume preserving crystalline mean curvature flow of convex sets in $\mathbb{R}^{N}$, J. Math. Pures Appl., 92, (2009), 499-527.

[6] G. Bellettini, M. Novaga and M. Paolini, Facet-breaking for three-dimensional crystals evolving by mean curvature, Interfaces and Free boundaries, 1 (1999), 39-55. 
[7] G. Bellettini, M. Novaga and M. Paolini, On a crystalline variational problem, part I: First variation and global $L^{\infty}$ regularity. Arch. Rational Mech. Anal., 157, 165-191 (2001).

[8] G. Bellettini, M. Novaga and M. Paolini, Characterization of facet-breaking for nonsmooth curvature flow in the convex case, Interfaces Free Bound., 3 (2001), 415-446.

[9] W.F.Berg, Crystal growth from solutions, Proc. Roy. Soc. London A, 164, (1938), 79-95.

[10] X.Chen and F.Reitich, Local existence and uniqueness of solutions to the Stefan problem with surface tension and kinetic undercooling, J. Math. Anal. Appl., 164 (1992), 350-362.

[11] M.Crandall, Viscosity solutions: a primer. Viscosity solutions and applications (Montecatini Terme, 1995), 1-43, Lecture Notes in Math., 1660, Springer, Berlin, 1997.

[12] J.Escher, J.Prüss, G.Simonett, Analytic solutions for a Stefan problem with GibbsThomson correction. J. Reine Angew. Math., 563, (2003), 1-52.

[13] T. Fukui and Y. Giga, Motion of a graph by nonsmooth weighted curvature, In: World congress of nonlinear analysts '92 (ed. V. Lakshmikantham), Walter de Gruyter, Berlin I, (1996), 47-56.

[14] M.-H. Giga, Y. Giga, Evolving graphs by singular weighted curvature, Arch. Rational Mech. Anal., 141 (1998), 117-198.

[15] M.-H. Giga, Y. Giga, On the role of kinetic and interfacial anisotropy in the crystal growth theory, Interfaces Free Bound., 15 (2013), 429-450.

[16] M.H. Giga, Y. Giga, P.Rybka, A comparison principle for singular diffusion equations with spatially inhomogeneous driving force, Arch. Ration. Mech. Anal., 211, (2014), 419-453. M.H. Giga, Y. Giga, P.Rybka, Erratum to: A comparison principle for singular diffusion equations with spatially inhomogeneous driving force for graphs, Arch. Ration. Mech. Anal., 212, (2014), 707.

[17] Y.Giga, P.Górka, P.Rybka, Nonlocal spatially inhomogeneous Hamilton-Jacobi equation with unusual free boundary, Discrete Contin. Dynam. Systems A, 26, (2010), 493-519.

[18] Y.Giga, P.Górka, P.Rybka, Evolution of regular bent rectangles by the driven crystalline curvature flow in the plane with a non-uniform forcing term, Adv. Differential Equations, 18, (2013), no. 3-4, 201-242.

[19] Y.Giga, P.Rybka, Berg's Effect, Adv. Math. Sci. Appl., 13, (2003), 625-637.

[20] Y.Giga, P.Rybka, Stability of facets of crystals growing from vapor, Discrete Contin. Dyn. Syst.., 14 (2006), no. 4, 689-706.

[21] Y.Giga, P.Rybka, Facet bending in the driven crystalline curvature flow in the plane, $J$. Geom. Anal., 18, (2008), 109-147.

[22] Y.Giga, P.Rybka, Facet bending driven by the planar crystalline curvature with a generic nonuniform forcing term, J. Diff. Eqs., 246, (2009), 2264-2303.

[23] M.E.Gurtin, Thermomechanics of Evolving Phase Boundaries in the Plane. Oxford Mathematical Monographs. The Clarendon Press Oxford University Press, New York, 1993.

[24] R.Kobayashi, Y.Giga, On anisotropy and curvature effects for growing crystals, Japan J. Indust. Appl. Math., 18(2), (2001), 207-230.

[25] A.Kubica, P.Rybka, Fine singularity analysis of solutions to the Laplace equation, to appear in Math. Meth. Appl. Sci.

[26] K.G.Libbrecht, The physics of snow crystals. Rep. Progr. Phys., 68(4) (2005), 855-895.

[27] S.Luckhaus, Solutions for the two-phase Stefan problem with the GibbsThomson law for the melting temperature, Eur. J. Appl. Math., 1 (2), (1990), 101-111.

[28] P.B.Mucha, On the Stefan problem with surface tension in the $L_{p}$ framework, Adv. Differential Equations, 10 (2005), no. 8, 861-900. 
[29] J.Prüss, G.Simonett, R.Zacher, Qualitative behavior of solutions for thermodynamically consistent Stefan problems with surface tension, Arch. Ration. Mech. Anal., 207 (2013), no. 2, 611-667.

[30] E.V.Radkevich, The Gibbs-Thomson correction and condition for the existence of classical solution of the modified Stefan problem, Soviet Math. Dokl., 43 (1991), 274-278.

[31] J.E.Taylor, J.W.Cahn, C.A.Handwerker, Overview No. 98. I - Geometric models of crystal growth. Acta Metall. Mater., 40(7), (1992), 1443-1474.

[32] A.Visintin, Models of phase transitions, Progress in Nonlinear Differential Equations and their Applications, 28. Birkhäuser Boston, Boston, MA, 1996. 